\title{
AN EULER-POINCARÉ FORMULA FOR A DEPTH ZERO BERNSTEIN PROJECTOR
}

\author{
DAN BARBASCH, DAN CIUBOTARU, AND ALLEN MOY
}

\begin{abstract}
Work of Bezrukavnikov-Kazhdan-Varshavsky uses an equivariant system of trivial idempotents of Moy-Prasad groups to obtain an EulerPoincaré formula for the $\mathrm{r}$-depth Bernstein projector. We establish an EulerPoincaré formula for natural sums of depth zero Bernstein projectors (which is often the projector of a single Bernstein component) in terms of an equivariant system of Peter-Weyl idempotents of parahoric subgroups $\mathcal{G}_{F}$ associated to a block of the reductive quotient $\mathcal{G}_{F} / \mathcal{G}_{F}^{+}$.
\end{abstract}

\section{INTRODUCTION}

The results expounded here are the merger of several themes in the representation theory of reductive p-adic groups. Suppose $k$ is a non-archimedean local field, and $\mathbf{G}$ is a connected reductive linear algebraic group defined over $k$. Let $\mathcal{G}=\mathbf{G}(k)$ denote the group of $k$-rational points, and let $\mathcal{B}=\mathcal{B}(\mathcal{G})$ denote the Bruhat-Tits building of $\mathcal{G}$.

Two themes introduced in the 1980s were Euler-Poincaré functions $[\mathrm{K}]$ and the Bernstein center $[\mathrm{B}, \mathrm{BD}$.

- When $\mathcal{G}$ is semisimple, simply connected, Kottwitz selected a set $\varphi$ of representatives for the orbits of $\mathcal{G}$ on the facets of $\mathcal{B}$, and defined an EulerPoincaré function $f_{\mathrm{EP}} \in C_{c}^{\infty}(\mathcal{G})$ (locally constant compactly supported functions on $\mathcal{G}$ ) as $f_{\mathrm{EP}}=\sum_{\sigma \in \varphi}(-1)^{\operatorname{dim}(\sigma)} \frac{1}{\operatorname{Stab}(\sigma)} \operatorname{sgn}_{\sigma}$ (see [K] for the description of the character $\operatorname{sgn} n_{\sigma}$ of $\operatorname{Stab}(\sigma)$ and other normalizations). It is obvious that changing the set $\varphi$ changes $f_{\mathrm{EP}}$, but not its orbital integrals. Kottwitz showed the usefulness of $f_{\mathrm{EP}}$ as a function to enter into the trace formula.

- The Bernstein center $\mathcal{Z}=\mathcal{Z}(\mathcal{G})$ of $\mathcal{G}$ is a commutative algebra (with unity). Its geometrical realization is as the algebra of $\mathcal{G}$-invariant essentially compact distributions on $\mathcal{G}$. A distribution is essentially compact if $\forall f \in C_{c}^{\infty}(\mathcal{G})$, the convolutions $D \star f$ and $f \star D$ are in $C_{c}^{\infty}(\mathcal{G})$. If $\left(\pi, V_{\pi}\right)$ is a smooth representation of $\mathcal{G}$, one can, by integration, canonically obtain an algebra representation $\pi_{\mathcal{Z}}: \mathcal{Z}(\mathcal{G}) \longrightarrow \operatorname{End}_{\mathcal{G}}\left(V_{\pi}\right)$. When $\pi$ is irreducible, by Schur's Lemma, each $\pi_{\mathcal{Z}}(D) \quad(D \in \mathcal{Z})$ is a scalar.

Received by the editors March 10, 2018 and in revised form, February 14, 2019.

2010 Mathematics Subject Classification. Primary 22E50, 22E35.

Key words and phrases. Bernstein center, Bernstein projector, Bruhat-Tits building, depth, distribution, equivariant system, essentially compact, Euler-Poincaré, idempotent, resolution.

The first author was partly supported by NSA grant H98230-16-1-0006.

The second author was partly supported by United Kingdom EPSRC grant EP/N033922/1.

The third author was partly supported by Hong Kong Research Grants Council grant CERG \#603813. 
Let $\widehat{\mathcal{G}}^{\text {sm }}$ denote the smooth dual (equivalence classes of smooth irreducible representations). We write the map $\{\pi\} \rightarrow \pi_{\mathcal{Z}}(D)$ as a map $\operatorname{Inf}_{D}: \widehat{\mathcal{G}}^{\mathrm{sm}} \longrightarrow \mathbb{C}$ given by $\operatorname{Inf}_{D}(\{\pi\})=\pi_{\mathcal{Z}}(D)$. The smooth dual is naturally topologized with the Fell topology, a non-Hausdorff topology. There is natural Hausdorff topological quotient $\Omega(\mathcal{G})$ of $\widehat{\mathcal{G}}^{\mathrm{sm}}$. The map $\operatorname{Inf}_{D}$ factors to a map $\Omega(\mathcal{G}) \longrightarrow \mathbb{C}$ (that we also denote as $\operatorname{Inf}_{D}$ ). The

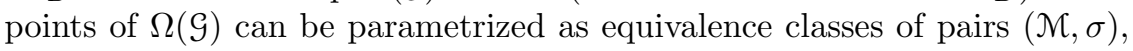
where $\mathcal{M}$ is a Levi subgroup of $\mathcal{G}$, and $\sigma$ is an irreducible cuspidal representation of $\mathcal{M}$. The equivalence relation comes from the adjoint action on the Levi component. The equivalence class of a pair $(\mathcal{M}, \sigma)$ is denoted $[\mathcal{M}, \sigma]$. For a fixed Levi subgroup $\mathcal{M}$, the complex group $X_{\text {un }}(\mathcal{M})$ of unramified characters acts on the set of elements $[\mathcal{M}, \sigma]$ by twisting the representation component (see $[\mathrm{B}, \mathrm{ChII}]$ ), and the orbit (denoted $\Omega([\mathcal{M}, \sigma])$ ) is a Bernstein component. Thus, $\Omega(\mathcal{G})$ is partitioned into Bernstein components. The component $\Omega([\mathcal{M}, \sigma])$ inherits a complex algebraic structure from $X_{\text {un }}(\mathcal{M})$. The restriction $\left(\operatorname{Inf}_{D}\right)_{\left.\right|_{\Omega}}$ of the function $\operatorname{Inf}_{D}$ to a Bernstein component $\Omega=\Omega([\mathcal{M}, \sigma])$ is a regular function. Let $\mathcal{R}(\Omega)$ denote the algebra of regular functions on $\Omega$. In $\left[\mathrm{BD}\right.$, it is shown that the map $\mathrm{FT}_{\Omega}: \mathcal{Z}(\mathcal{G}) \rightarrow \mathcal{R}(\Omega)$ defined as $\operatorname{FT}_{\Omega}(D)=\left(\operatorname{Inf}_{D}\right)_{\left.\right|_{\Omega}}$ is a surjective algebra homomorphism, and there is an ideal $\mathcal{I}_{\Omega}$ of $\mathcal{Z}(\mathcal{G})$ so that $\mathrm{FT}_{\Omega}$ on $\mathcal{I}_{\Omega}$ is an isomorphism, while $\mathrm{FT}_{\Omega}$ on $\mathcal{I}_{\Omega^{\prime}}\left(\Omega \neq \Omega^{\prime}\right)$ is zero. So, $\mathcal{Z}(\mathcal{G})$ is a product of the ideals $\mathcal{I}_{\Omega}$. The unique element $P(\Omega) \in \mathcal{Z}(\mathcal{G})$ satisfying $\left(\operatorname{Inf}_{P(\Omega)}\right)_{\Omega_{\Omega^{\prime}}}=\delta_{\Omega, \Omega^{\prime}}$ is called the projector of the Bernstein component $\Omega$. At the time (1980s) extremely little was known explicitly about the distribution $P(\Omega)$. The most illuminating result at that time was a 1976 result of Deligne $[\mathrm{Dn}]$. Suppose $\mathcal{G}$ has compact center. Then, Deligne's result is: The support of the character of an irreducible cuspidal representation is in the set of compact elements (those elements which belong to a compact subgroup) of $\mathcal{G}$. This was extended by Dat $\mathrm{Dt}$ in 2003 to the statement that the projector $P(\Omega)$ of a Bernstein component $\Omega$ has support in the compact elements of $\mathcal{G}$.

In the 1990s, exploitation of the Bruhat-Tits building achieved advances in two directions.

- Moy-Prasad [MPa, $\mathrm{MPb}$ used points in $\mathcal{B}$ to define subgroups of $\mathcal{G}$ and lattices of $\mathfrak{g}=\operatorname{Lie}(\mathcal{G})$ which satisfy descent properties. In particular, these subgroups and lattices allow one to attach to any irreducible smooth representation $\left(\pi, V_{\pi}\right)$ a non-negative rational number $\rho(\pi)$ called the depth. The application of the parabolic induction functor or the Jacquet functor to an irreducible representation, yields a representation whose constituents all have the same depth as the input. Thus, all the irreducible representations attached to a Bernstein component $\Omega$ have the same depth, i.e., one can define the depth $\rho(\Omega)$ of a component $\Omega$. It is clear from their definitions that the Moy-Prasad groups and lattices are $\mathcal{G}$-equivariant objects of $\mathcal{B}$.

- Schneider-Stuhler SS attached to a smooth representation $\left(\pi, V_{\pi}\right)$ a $\mathcal{G}$ equivariant coefficient system $\gamma_{e}\left(V_{\pi}\right)$. To a facet $F$ of the building with parahoric subgroup $U_{F}$ (notation that of Schneider-Stuhler), and positive integer $e$, they define a subgroup $U_{F, e}$ (which, if $y$ is a generic point of $F$, is in fact the Moy-Prasad group $\left.\mathcal{G}_{y, e}\right)$. The coefficient system is $\gamma_{e}\left(V_{\pi}\right)(F):=$ $V_{\pi}^{U_{F, e}}$. The space of global sections with compact support in the facets of 
a given dimension $i$ is a projective smooth representation of $\mathcal{G}$. SchneiderStuhler used the standard boundary map to get a complex, and under suitable circumstances they proved the important result that this complex is a projective resolution of $V_{\pi}$.

No serious attempt was made in the 1990s to synthesize these two directions together.

An important development made by Meyer-Solleveld [MS in 2010 was to replace the coefficient systems of Schneider-Stuhler with idempotent operators $e_{\sigma}(\sigma$ a facet in $\mathcal{B})$ in $\operatorname{End}_{\mathbb{C}}\left(V_{\pi}\right)$. The situation of Schneider-Stuhler can be recovered from Meyer-Solleveld by taking $e_{\sigma}$ to be the idempotent which projects to the space of $U_{\sigma, e}$-fixed vectors. A key aspect of their idempotent approach is that the chain complex on $\mathcal{B}$ attached to the idempotents has the property that its restriction to a finite polysimplicial convex subcomplex $\Sigma$ of $\mathcal{B}$ is a resolution of the vector space $\sum_{x \in \Sigma^{\circ}} e_{x}\left(V_{\pi}\right)$, where $\Sigma^{\circ}$ is the set of vertices in $\Sigma$. Most importantly, under certain assumptions on the system of idempotents (see [MS]), they showed the operator $\sum_{\sigma \in \Sigma}(-1)^{\operatorname{dim}(\sigma)} e_{\sigma}$ is idempotent and projects $V_{\pi}$ to $\sum_{x \in \Sigma^{\circ}} e_{x}\left(V_{\pi}\right)$.

The work of Bezrukavnikov-Kazhdan-Varshavsky BKV] in 2016 linked the Schneider-Stuhler and Meyer-Solleveld theme to Bernstein projectors. For ease of exposition of their work, we assume $\mathcal{G}$ is absolutely quasisimple (see BKV] for their more general situation). Suppose $r \geq 0$ is a rational number (write $r=\frac{a}{b}$ with $a, b \in \mathbb{N}, \operatorname{gcd}(a, b)=1)$. They modify the Meyer-Solleveld approach:

(i) They take a natural refinement of the simplicial structure on an apartment subdividing the space between two consecutive parallel affine hyperplanes into $b$ congruent regions. This refinement of the simplicial structure has the feature that the filtration subgroups $\mathcal{G}_{y, r}$ and $\mathcal{G}_{y, r^{+}}$(recall $\mathcal{G}_{y, r^{+}}:=$ $\left.\bigcap_{t>r} \mathcal{G}_{y, t}\right)$ are constant on the interiors of the new facets. For a new facet $F$, define $\mathcal{G}_{F, r+}$ to be $\mathcal{G}_{y, r+}$ of an interior point $y$.

(ii) They replaced the idempotents in $\operatorname{End}\left(V_{\pi}\right)$ with idempotents

$$
e_{F, r^{+}}=\frac{1}{\operatorname{meas}\left(\mathcal{G}_{F, r^{+}}\right)} 1_{\mathcal{G}_{F, r^{+}}}
$$

in the Hecke algebra $\mathcal{H}(\mathcal{G}) .\left(\mathcal{H}(\mathcal{G})\right.$ is $C_{c}^{\infty}(\mathcal{G})$ together with a choice of Haar measure.)

(iii) They considered an increasing family $\Sigma_{n} \quad(n \in \mathbb{N})$ of finite convex subcomplexes whose union is the entire building.

The resulting sums $\sum_{F \in \Sigma_{n}}(-1)^{\operatorname{dim}(F)} e_{F, r^{+}}$have limit the depth $r$ Bernstein projector

$$
P_{r}=\sum_{\rho(\Omega) \leq r} P(\Omega)
$$

and furthermore, as a distribution, $P_{r}$ has a presentation as an Euler-Poincaré sum $P_{r}=\sum_{F \subset \mathcal{B}}(-1)^{\operatorname{dim}(F)} e_{F, r^{+}}$.

Here, under the condition that the $k$-group $\mathbf{G}$ is absolutely quasisimple, we further develop the new direction of [BKV]. We establish an Euler-Poincaré presentation of the projector for an arbitrary Bernstein component of depth zero. The condition that $\mathbf{G}$ is absolutely quasisimple has the simplifying convenience that the Bruhat-Tits building $\mathcal{B}(\mathcal{G})(\mathcal{G}=\mathbf{G}(k))$ is a simplicial complex.

When $\mathcal{L}$ is a Levi subgroup of $\mathcal{G}$, let $\mathcal{B}_{\mathcal{G}}(\mathcal{L})$ be the union of the apartments $\mathcal{A}(\mathcal{S})$ as $\mathcal{S}$ runs over the maximal split tori in $\mathcal{L}$. The space $\mathcal{B}_{\mathcal{G}}(\mathcal{L})$ is the extended 
building of $\mathcal{L}$. Suppose $\Omega([\mathcal{M}, \pi])$ is a depth zero Bernstein component. It is known from $[\mathrm{MPb}$ that there exists a cuspidal pair $(F, \sigma)$ consisting of:

(i) a facet $F$ in $\mathcal{B}_{\mathcal{G}}(\mathcal{M})$ satisfying $\left(\mathcal{M} \cap \mathcal{G}_{F}\right) /\left(\mathcal{M} \cap \mathcal{G}_{F}^{+}\right)=\mathcal{G}_{F} / \mathcal{G}_{F}^{+}$,

(ii) an irreducible representation $\sigma$ of $\mathcal{M}_{F}:=\left(\mathcal{M} \cap \mathcal{G}_{F}\right)$ inflated from a cuspidal representation of the finite field group $\left(\mathcal{M} \cap \mathcal{G}_{F}\right) /\left(\mathcal{M} \cap \mathcal{G}_{F}^{+}\right)=\mathcal{G}_{F} / \mathcal{G}_{F}^{+}$,

so that $\pi=\mathrm{c}-\operatorname{Ind}_{\mathcal{F}_{F}}^{\mathcal{M}}(\tau)$, where $\mathcal{F}_{F}$ is the normalizer subgroup $N_{\mathcal{M}}\left(\mathcal{M}_{F}\right)$, and $\tau$ is an extension of $\sigma$. Here, $\mathcal{G}_{F}^{+}$is the maximal normal pro-p-subgroup of $\mathcal{G}_{F}$. If $y$ is a generic point of $F$, so $\mathcal{G}_{F}=\mathcal{G}_{y, 0}$, then $\mathcal{G}_{F}^{+}=\mathcal{G}_{y, 0^{+}}$. The relation $\left(\mathcal{M} \cap \mathcal{G}_{F}\right) /(\mathcal{M} \cap$ $\left.\mathcal{G}_{F}^{+}\right)=\mathcal{G}_{F} / \mathcal{G}_{F}^{+}$means $\sigma$ is also canonically a representation of $\mathcal{G}_{F}$.

If $\tau^{\prime}$ is any extension of $\sigma$ to $\mathcal{F}_{F}$, then $\pi^{\prime}=\mathrm{c}-\operatorname{Ind}_{\mathcal{F}_{F}}^{\mathcal{M}}\left(\tau^{\prime}\right)$ is an irreducible cuspidal representation of $\mathcal{M}$ and any irreducible smooth representation containing $\sigma$ has this form (see $\mathrm{MPb}$ ). Let $\Omega(F, \sigma)$ be the (finite) union of the Bernstein components $\Omega([\mathcal{M}, \pi])$ in which the restriction of $\pi$ to $\mathcal{M}_{F}$ contains $\sigma$. Define

$$
P(F, \sigma):=\text { Bernstein projector to } \Omega(F, \sigma) \text {. }
$$

Let $\mathcal{N} \mathcal{V}$ be a parabolic subgroup containing $\mathcal{M}$, and set $V_{\pi}=\operatorname{Ind}_{\mathcal{M} \mathcal{V}}^{\mathcal{G}}(\pi)$. If $E$ is any facet of $\mathcal{B}$, it follows from $\mathrm{MPb}$ that a necessary and sufficient condition for the invariants $V_{\pi}^{\mathcal{G}_{E}^{+}}$to be non-zero is the existence of a facet $F^{\prime}$ so that:

(i) $F^{\prime} \supset E$,

(ii) $F^{\prime}$ is associate to $F$, i.e., there exists $g \in \mathcal{G}$, so that $\left(\mathcal{G}_{F^{\prime}} \cap \mathcal{G}_{g F}\right)$ surjects onto both $\mathcal{G}_{F^{\prime}} / \mathcal{G}_{F^{\prime}}^{+}$and $\mathcal{G}_{g F} / \mathcal{G}_{g F}^{+}$. Note that we then have an identification $\mathcal{G}_{F^{\prime}} / \mathcal{G}_{F^{\prime}}^{+}=\left(\mathcal{G}_{F^{\prime}} \cap \mathcal{G}_{g F}\right) /\left(\mathcal{G}_{F^{\prime}}^{+} \cap \mathcal{G}_{g F}^{+}\right)=\mathcal{G}_{g F} / \mathcal{G}_{g F}^{+}$.

Let $\mathcal{E}(\sigma, E)$ denote the set of representations $\rho \in \widehat{\mathcal{G}_{E} / \mathcal{G}_{E}^{+}}$which appear in $V_{\pi}^{\mathcal{S}_{E}^{+}}$. With the obvious identifications and inclusions, this set is those $\rho \in \widehat{\mathcal{G}_{E} / \mathcal{G}_{E}^{+}}$whose restriction to $\mathcal{G}_{F^{\prime}} / \mathcal{G}_{F^{\prime}}^{+}\left(=\mathcal{G}_{g F} / \mathcal{G}_{g F}^{+}\right)$contains the representation $\operatorname{Ad}(g) \sigma$.

For the Bernstein component $\Omega([\mathcal{N}, \pi])$ if $\left(F_{1}, \sigma_{1}\right)$ and $\left(F_{2}, \sigma_{2}\right)$ are as above, then $F_{1}$ and $F_{2}$ are associated by some $g \in \mathcal{G}$ with $\operatorname{Ad}(g) \sigma_{1}=\sigma_{2}$.

Fix a cuspidal pair $(F, \sigma)$. We define idempotents as follows:

$$
e_{E}= \begin{cases}0 & \text { when } V_{\pi}^{\mathcal{S}_{E}^{+}}=\{0\}, \\ \frac{1}{\operatorname{meas}\left(\mathcal{G}_{E}^{+}\right)} \sum_{\rho \in \mathcal{E}(\sigma, E)} \operatorname{deg}(\rho) \Theta_{\rho} & \text { when } V_{\pi}^{\mathcal{G}_{E}^{+}} \neq\{0\} .\end{cases}
$$

Since we have fixed $(F, \sigma)$, we suppress it in the notation $e_{E}$. We call $e_{E}$ the Peter-Weyl idempotent. This defines a $\mathcal{G}$-equivariant system of idempotents on $\mathcal{B}$. The first and third authors established in earlier work that $e_{E}=P(F, \sigma) \star e_{\mathcal{G}_{E}^{+}}$. Once the $\mathcal{G}$-equivariant system of idempotents is in hand, it remains to show (see Theorem 5.2.5, Corollary 5.2.6, and Theorem 6.1.3) the following.

Theorem. Suppose $\mathbf{G}$ is a connected absolutely quasisimple k-group. Let $\mathcal{G}=$ $\mathbf{G}(k)$, and let $\mathcal{B}=\mathcal{B}(\mathcal{G})$ be the Bruhat-Tits building. Suppose $(F, \sigma)$ is a cuspidal

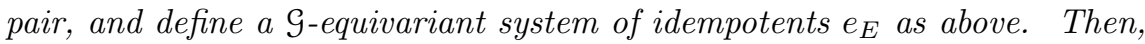

- The Euler-Poincaré sum

$$
P=\sum_{L \subset \mathcal{B}(\mathcal{G})}(-1)^{\operatorname{dim}(L)} e_{L}
$$


over the facets of $\mathcal{B}(\mathcal{G})$ defines a $\mathcal{G}$-invariant essentially compact distribution.

- The distribution $P$ is the projector $P(F, \sigma)$.

We note, for the Iwahori component (smooth irreducible representations with non-zero Iwahori fixed vectors), the (Iwahori) Peter-Weyl idempotent $e_{F}$ of a facet $F$ is the sum of the character idempotents of those irreducible representations of the finite field group $\mathcal{G}_{F} / \mathcal{G}_{F}^{+}$which have a non-zero Iwahori fixed vector, i.e., a Borel fixed vector.

We sketch the argument to show the Euler-Poincaré infinite sum defines an essentially compact distribution. We fix a chamber $C_{0}$, and define the convex ball $\operatorname{Ball}\left(C_{0}, m\right)$ to be the simplicial subcomplex which is the union of all chambers whose Bruhat length from $C_{0}$ is at most $m$. The union of these balls obviously exhaust $\mathcal{B}$, and $\operatorname{Ball}\left(C_{0},(m+1)\right)$ is obtained by adding chambers of Bruhat length $(m+1)$ to $\operatorname{Ball}\left(C_{0}, m\right)$. If $D$ has Bruhat length $(m+1)$, let $\mathcal{C}(D)$ be the set of facets of $D$ which are not already in $\operatorname{Ball}\left(C_{0}, m\right)$. We note that if $k$ is the number of faces of $D$ in $\mathcal{C}(D)$, then $\#(\mathcal{C}(D))=2^{k}$. If $J$ is any open compact subgroup of $\mathcal{G}$, we show the convolution

$$
\text { Con }=\left(\sum_{E \in \mathcal{C}(D)}(-1)^{\operatorname{dim}(E)} e_{E}\right) \star e_{J}
$$

vanishes once $m$ is sufficiently large, say $m \geq N$. Hence,

$\sum_{E \in \operatorname{Ball}\left(C_{0}, n\right)}(-1)^{\operatorname{dim}(E)} e_{E} \star e_{J}=\sum_{E \in \operatorname{Ball}\left(C_{0}, N\right)}(-1)^{\operatorname{dim}(E)} e_{E} \star e_{J} \quad$ for all $n \geq N$.

We deduce that the infinite Euler-Poincaré sum defines a $\mathcal{G}$-invariant essentially compact distribution $P$. A slight modification of the argument then allows us to establish $P$ is the projector $P(\Omega)$ to the component $\Omega$.

We briefly explain here why the convolution Con vanishes when $m$ is sufficiently large. In the set $\mathcal{C}(D)$, there is a minimal facet $D_{+}$contained in all the other facets. This means $\mathcal{G}_{D_{+}} \supset \mathcal{G}_{E} \supset \mathcal{G}_{E}^{+} \supset \mathcal{G}_{D_{+}}^{+}$holds for all $E \in \mathcal{C}(D)$, and consequently $\mathcal{G}_{E} / \mathcal{G}_{D_{+}}^{+}$is a parabolic subgroup in $\mathcal{G}_{D_{+}} / \mathcal{G}_{D_{+}}^{+}$. A convolution vanishing result is established in the finite field group $\mathcal{G}_{D_{+}} / \mathcal{G}_{D_{+}}^{+}$, which when the Bruhat length is sufficiently large implies the vanishing of Con.

We briefly outline the presentation of results. In section 2, we introduce notation, and prove preliminary results on facets in the Bruhat-Tits building. A key result (Proposition 2.5.5) on facets is proved in the last subsection. Section 3 is a review of basic results on representations of connected reductive groups over a finite field that follows from Harish-Chandra's philosophy of cusp forms [HC]. In sections 4 and 5 we prove the main result when $\mathbf{G}$ is split. In section 6 , we indicate the modifications that adapt the proofs of sections 4 and 5 to the non-split setting.

We make two remarks about our idempotent systems and the work in [MS] and BKV].

- In [MS, axioms for a system of idempotents are introduced, and it is established that an Euler-Poincaré sum over a finite convex subcomplex $\Sigma$ is a certain explicit projection operator. They then take a limit and obtain elements of the Bernstein center (their terminology is central idempotent in the multiplier algebra). They provide examples related to $\mathrm{GL}(m)$, the work 
of Schneider-Stuhler [SS, and cases associated to certain supercuspidal representations.

- When $r$ is a rational number, in [BKV], it is established directly that EulerPoincaré sums of idempotents of the trivial representation of Moy-Prasad groups $\mathcal{G}_{x, r^{+}}$provide a presentation of the depth $r$ Bernstein projector $P_{r}$. In particular, when $r$ is not integral, a simplicial refinement of the building is required. These provide interesting examples of Euler-Poincaré sum presentations (for natural finite sums) of Bernstein projectors.

In our work here, for an individual Bernstein projector $P_{\Omega}$ of depth zero, we define a system of Peter-Weyl idempotents and show directly the Euler-Poincaré sum of these idempotents provides a presentation of $P_{\Omega}$. If we fix a facet $F$ and sum over the Bernstein components of depth zero, then by Harish-Chandra's philosophy of cusp forms, and the Peter-Weyl Theorem, the sum is the inflation of the regular representation of $\mathcal{G}_{F} / \mathcal{G}_{F}^{+}$, i.e., the idempotent $\frac{1}{\operatorname{meas}\left(\mathcal{G}_{F}^{+}\right)} 1_{\mathcal{G}_{F}^{+}}$. This is the EulerPoincaré presentation of $P_{0}$ in $[\mathrm{BKV}]$. We mention it can be shown a posteriori our system of idempotents satisfy the axioms in $\mathrm{MS}$.

The evidence provided by the Euler-Poincaré formula for $P_{0}$ and individual depth zero projectors, leads to the expectation there should be an Euler-Poincaré formula for suitable combinations of Bernstein projectors. In the extreme case of a single Bernstein component and positive depth, the equivariant data should involve refinements of the unrefined minimal K-types of $[\mathrm{MPa}, \mathrm{MPb}$.

\section{Notation, Review, AND RESUlts on FACETS IN THE BRUHAT-TITS BUILDING}

2.1. Notation. Suppose $k$ is a non-archimedean local field. Denote by $\mathfrak{O}_{k}, \wp_{k}$, and $\mathbb{F}_{q}=\mathfrak{O}_{k} / \wp_{k}$, respectively, the ring of integers, prime ideal, and residue field of $k$. Let $\mathbf{G}$ be a connected reductive linear algebraic group defined over $k$. If $\mathbf{H}$ is a $k$-subgroup of $\mathbf{G}$, we write $\mathcal{H}$ for the group of $k$-rational points of $\mathbf{H}$, e.g., $\mathcal{G}=\mathbf{G}(k)$. For convenience, we assume $\mathbf{G}$ is $k$-split and absolutely quasisimple. Set $\ell=\operatorname{rank}(\mathbf{G})$. Let $\mathcal{B}=\mathcal{B}(\mathcal{G})$ be the reduced Bruhat-Tits building of $\mathcal{G}$. Let $\mathbf{S}$ be a maximal $k$-split torus of $\mathbf{G}$, and let $\mathcal{A}=\mathcal{A}(\mathcal{S})$ be the apartment associated to $\mathcal{S}=\mathbf{S}(k) \subset \mathcal{G}$. Our hypotheses on $\mathbf{G}$ (split, quasisimple) mean the apartments are simplicial complexes, and hence $\mathcal{B}$ is too. The group $\mathcal{G}$ acts transitively on the chambers $\left(\ell\right.$-simplices) of $\mathcal{B}$. The choice of a hyperspecial point $x_{0} \in \mathcal{A}$ corresponds to the choice of a Chevalley basis for the Lie algebra $\mathfrak{g}$ of $\mathcal{G}$.

Let $\Phi=\Phi(\mathcal{S}$ ) be the roots (of $\mathcal{G}$ ) with respect to $\mathcal{S}$, and let $\Psi=\Psi(\mathcal{A})$ be the system of affine roots on $\mathcal{A}$. If $\alpha$ (resp., $\psi$ ) is a root (resp., affine root), set $\mathcal{U}_{\alpha}$ (resp., $X_{\psi}$ ) to be the associated root (resp., affine root) group. If $\Phi^{+}$is any set of positive roots of $\Phi$, let $\Delta$ denote the simple roots subset of $\Phi^{+}$.

- Fix a Borel subgroup $\mathbf{B} \supset \mathbf{S}$ of $\mathbf{G}$, and let $\Phi_{\mathcal{B}}^{+}=\Phi(\mathcal{S}, \mathcal{B})$ denote the set of positive roots with respect to $\mathcal{B}$, and $\Delta_{\mathcal{B}}=\left\{\alpha_{1}, \ldots, \alpha_{\ell}\right\}$ the simple roots.

(i) Let $\psi_{i} \quad(1 \leq i \leq \ell)$ be the affine roots so that $\operatorname{grad}\left(\psi_{i}\right)=\alpha_{i}$, and $\psi\left(x_{0}\right)=0$.

(ii) Let $\psi_{0}$ be the affine root so that $\operatorname{grad}\left(\psi_{0}\right)$ is the negative of the highest root, and $\psi_{0}\left(x_{0}\right)=1$ (we have normalized the value group [T, $\left.\S 0.2\right]$ to be $\mathbb{Z})$. 
- Set

$$
\begin{aligned}
\mathcal{S}:= & \left\{x \in \mathcal{A} \mid \psi_{i}(x)>0 \quad \forall 1 \leq i \leq \ell\right\} \\
& \text { the positive Weyl chamber in } \mathcal{A} \text { with respect to } x_{0} \text { and } \Phi^{+}, \\
\mathcal{S}_{0}:= & \left\{x \in \mathcal{A} \mid \psi_{i}(x)>0 \quad \forall 0 \leq i \leq \ell\right\} .
\end{aligned}
$$

Note $\mathcal{S}_{0} \subset \mathcal{S}$, and its closure $\overline{\mathcal{S}_{0}}$ is a chamber (affine Weyl chamber) in $\mathcal{A}$, and with respect to $\mathcal{S}_{0}$, the sets

$$
\Psi^{+}=\left\{\psi \in \Psi(\mathcal{A}) \mid \psi(x)>0 \forall x \in \mathcal{S}_{0}\right\} \quad \text { and } \quad \Delta_{0}=\left\{\psi_{0}, \psi_{1}, \ldots, \psi_{\ell}\right\}
$$

are the positive affine roots, and the simple affine roots, respectively. The affine roots $\Psi(\mathcal{A})$ are integer combinations $\psi=\sum n_{i} \psi_{i}$ satisfying $\operatorname{grad}(\psi)$ $\in \Phi$.

- For $x \in \mathcal{B}$, and $r \geq 0$, let $\mathcal{G}_{x, r}$ be the Moy-Prasad subgroup associated to $x$ and $r$.

- We fix a Haar measure on $\mathcal{G}$. If $J$ is an open compact subgroup of $\mathcal{G}$, we define $e_{J}$ to be the idempotent:

$$
e_{J}(x):=\frac{1}{\operatorname{meas}(J)} \begin{cases}1 & \text { if } x \in J \\ 0 & \text { otherwise }\end{cases}
$$

2.2. A simplex lemma. We recall and designate some nomenclature. An $\ell$ dimensional simplex $D$ is the convex closure of a set Vert $=\left\{v_{0}, v_{1}, \ldots, v_{\ell}\right\}$ of $(\ell+1)$ points in an affine space so that $v_{1}-v_{0}, v_{2}-v_{0}, \ldots, v_{\ell}-v_{0}$ are linearly independent. For a non-empty subset $K \subset$ Vert with $(k+1)$ elements, we designate:

- $\operatorname{facet}(K):=$ convex closure of $K$. It is a $k$-facet of $D$.

- The convex set

$$
\operatorname{recess}(K):=\operatorname{facet}(K) \backslash \bigcup_{L \subsetneq K} \operatorname{facet}(L) .
$$

The recess is the interior of facet $(K)$ when $k \geq 1$ and equal to facet $(K)$ for $k=0$. A useful feature of recesses is that the simplex $D$ is partitioned by them, and there is a one-to-one correspondence from recesses to facets, namely the process of taking the closure. For convenience, when $E$ is a facet of $D$, we write recess $(E)$ for the recess whose closure is $E$.

- It is elementary that:

(i) the number of $k$-facets contained in a $j$-facet $(j \geq k)$ is $\left(\begin{array}{l}j+1 \\ k+1\end{array}\right)$,

(ii) the total number of facets is $2^{\ell+1}-1$.

A face of $D$ is, by definition, the convex closure of $\ell$ points of $V$, i.e., a maximal proper facet of $D$.

- If $F$ is a facet in $\mathcal{B}(\mathcal{G})$, and $y \in \operatorname{recess}(F)$, let $\mathcal{G}_{F}$ denote the parahoric subgroup $\mathcal{G}_{y, 0}$, and let $\mathcal{G}_{F}^{+}=\mathcal{G}_{y, 0^{+}}$.

Lemma 2.2.1. Suppose $D$ is an $\ell$-dimensional simplex, and $\mathcal{F}$ is a non-empty collection of faces of $D$. Set $m=\#(\mathcal{F})$. Then

(i) The union $\mathcal{P}=\bigcup_{F \in \mathcal{F}} F$ is a simplicial complex inside D. The number of $k$-facets of $D$ in $\mathcal{P}$ is

$$
\sum_{r=1}^{m}(-1)^{(r-1)}\left(\begin{array}{c}
\ell+1-r \\
k+1
\end{array}\right)\left(\begin{array}{c}
m \\
r
\end{array}\right) .
$$


(ii) Let $\mathcal{C}$ be the facets of $D$ occurring in the complement of $\mathcal{P}$. Then:

(ii.1) $A$ facet in $\mathcal{C}$ has codimension at most $(\ell+1-m)$.

(ii.2) The number of facets of codimension $j$ is $\left(\begin{array}{c}\ell+1-m \\ j\end{array}\right)$.

(ii.3) The total number of facets in $\mathcal{C}$ is $2^{\ell+1-m}$.

We note: (i) when $\mathcal{F}$ is all the $\ell+1$ faces, then $\mathcal{C}$ consists of $D$, (ii) when $\mathcal{F}$ is all but one of the faces, then $\mathcal{C}$ consists of two elements (the remaining face, and $D$ itself), (iii) when $\mathcal{F}=\{F\}$ is a single face $F$, then $\mathcal{C}$ consists of all the facets not contained in $F$.

Proof. The proof of (i) is based on inclusion and exclusion. Suppose $\Sigma$ is the closure of a $j$-facet in the union $\mathcal{P}$. For $k \leq j$, the number of $k$-facets in $\Sigma$ is $\left(\begin{array}{l}j+1 \\ k+1\end{array}\right)$. The intersection of $r$ distinct face closures is the closure of a unique $(\ell-r)$-facet, e.g., a single face is an $(\ell-1)$-facet. This $(\ell-r)$-facet has $\left(\begin{array}{c}\ell-r+1 \\ k+1\end{array}\right) k$-facets in its closure. By the principle of inclusion and exclusion, the number of $k$-facets in the union $\mathcal{P}$ is the stated

$$
\sum_{r=1}^{m}(-1)^{(r-1)}\left(\begin{array}{c}
\ell+1-r \\
k+1
\end{array}\right)\left(\begin{array}{c}
m \\
r
\end{array}\right) .
$$

To prove statement (ii), we consider the sum obtained by extending the index $r$ to $r=0$, i.e., the sum

$$
\sum_{r=0}^{m}(-1)^{(r-1)}\left(\begin{array}{c}
\ell+1-r \\
k+1
\end{array}\right)\left(\begin{array}{c}
m \\
r
\end{array}\right) .
$$

This is $(-1)^{(\ell-k+1)}$ times the coefficient of $x^{(\ell-k)}$ in the power series expansion of $\frac{1}{(1+x)^{(k+2)}}(1+x)^{m}$.

When $m \geq(k+2)$, the power series is the polynomial $(1+x)^{((m-2)-k)}$, and the coefficient of $x^{(\ell-k)}$ is zero; so,

$$
\left(\begin{array}{l}
\ell+1 \\
k+1
\end{array}\right)=\sum_{r=1}^{m}(-1)^{(r-1)}\left(\begin{array}{c}
\ell+1-r \\
k+1
\end{array}\right)\left(\begin{array}{c}
m \\
r
\end{array}\right) .
$$

In particular, all $k$-facets of $D$ are in the union $\mathcal{P}$, so none are in $\mathcal{C}$.

When $m<(k+2)$, the coefficient of $x^{(\ell-k)}$ in the power series expansion of $\frac{1}{(1+x)^{((k+2)-m)}}$ is $(-1)^{(\ell-k)}\left(\begin{array}{c}\ell+1-m \\ k+1-m\end{array}\right)$. Thus,

$$
\sum_{r=1}^{m}(-1)^{(r-1)}\left(\begin{array}{c}
\ell+1-r \\
k+1
\end{array}\right)\left(\begin{array}{c}
m \\
r
\end{array}\right)=\left(\begin{array}{l}
\ell+1 \\
k+1
\end{array}\right)-\left(\begin{array}{c}
\ell+1-m \\
k+1-m
\end{array}\right) .
$$

Thus, the number of $k$-facets (of $D)$ in $\mathcal{C}$ is $\left(\begin{array}{c}\ell+1-m \\ k+1-m\end{array}\right)=\left(\begin{array}{c}\ell+1-m \\ \ell-k\end{array}\right)$. The integer $j=\ell-k$ is the codimension.

Suppose $D$ is a chamber of $\mathcal{B}$, and $\mathcal{F}$ is a non-empty collection of $m$ faces of $D$. Let $W=\left\{E_{1}, E_{2}, \ldots, E_{(\ell+1-m)}\right\}$ be the faces of $D$ complementary to the faces in $\mathcal{F}$. Then, the facets in the complement $\mathcal{C}$ of codimension $j$ can be described as follows: Given a subset $Y \subset W$ of $j$ faces, let

$$
F(Y)=\left(\bigcap_{E \in Y} E\right) \text { a }(\ell-j) \text {-facet } .
$$

When $Y=\emptyset$, we use the convention $F(\emptyset)=D$; so, $\mathcal{G}_{F(\emptyset)}=\mathcal{G}_{D}$. Clearly, all the $2^{(\ell+1-m)}$ facets in $\mathcal{C}$ are obtained in this fashion. If $(\emptyset \subset) Y_{1} \subset Y_{2} \subset W$, 
then $D \supset F\left(Y_{1}\right) \supset F\left(Y_{2}\right) \supset F(W)$; so, $\mathcal{G}_{D} \subset \mathcal{G}_{F\left(Y_{1}\right)} \subset \mathcal{G}_{F\left(Y_{2}\right)} \subset \mathcal{G}_{F(W)}$, and $\mathcal{G}_{D}^{+} \supset \mathcal{G}_{F\left(Y_{1}\right)}^{+} \supset \mathcal{G}_{F\left(Y_{2}\right)}^{+} \supset \mathcal{G}_{F(W)}^{+}$. The quotient $\mathcal{B}=\mathcal{G}_{D} / \mathcal{G}_{F(W)}^{+}$is a Borel subgroup of the finite field group $\mathcal{G}=\mathcal{G}_{F(W)} / \mathcal{G}_{F(W)}^{+}$. The parahoric subgroups which fix the $2^{(\ell+1-m)}$ facets of Lemma 2.2 .1 part (ii.3) corresponds to the standard parabolic subgroups of $\mathcal{G}$ which contain the Borel subgroup $\mathcal{B}$.

2.3. Bruhat height. We fix an apartment $\mathcal{A}=\mathcal{A}(\mathcal{S})$ of the building, and a chamber $C_{0}$ in $\mathcal{A}$. Let $\mathcal{S}_{\mathrm{c}}$ denote the maximal bounded (compact) subgroup of $\mathcal{S}$. We recall that the normalizer $\mathcal{N}=N_{\mathcal{G}}(\mathcal{S})$ of $\mathcal{S}$ acts on $\mathcal{A}$, with action kernel equal to $\mathcal{S}_{\mathrm{c}}$, i.e., the action factors through the extended affine Weyl group

$$
W^{\mathrm{a}}:=N_{\mathcal{G}}(\mathcal{S}) / \mathcal{S}_{\mathrm{c}} \text {. }
$$

For $n \in W^{\mathrm{a}}$, let $\ell_{\mathrm{Bru}}(n)$ denote the Bruhat length of $n$. If $D=n . C_{0}$, we define the Bruhat height of $D$ with respect to $C_{0}$ as:

$$
\operatorname{ht}_{C_{0}}(D):=\ell_{\mathrm{Bru}}(n) .
$$

Figure 1 illustrates the height function ht $C_{0}$ for $C_{2}$.

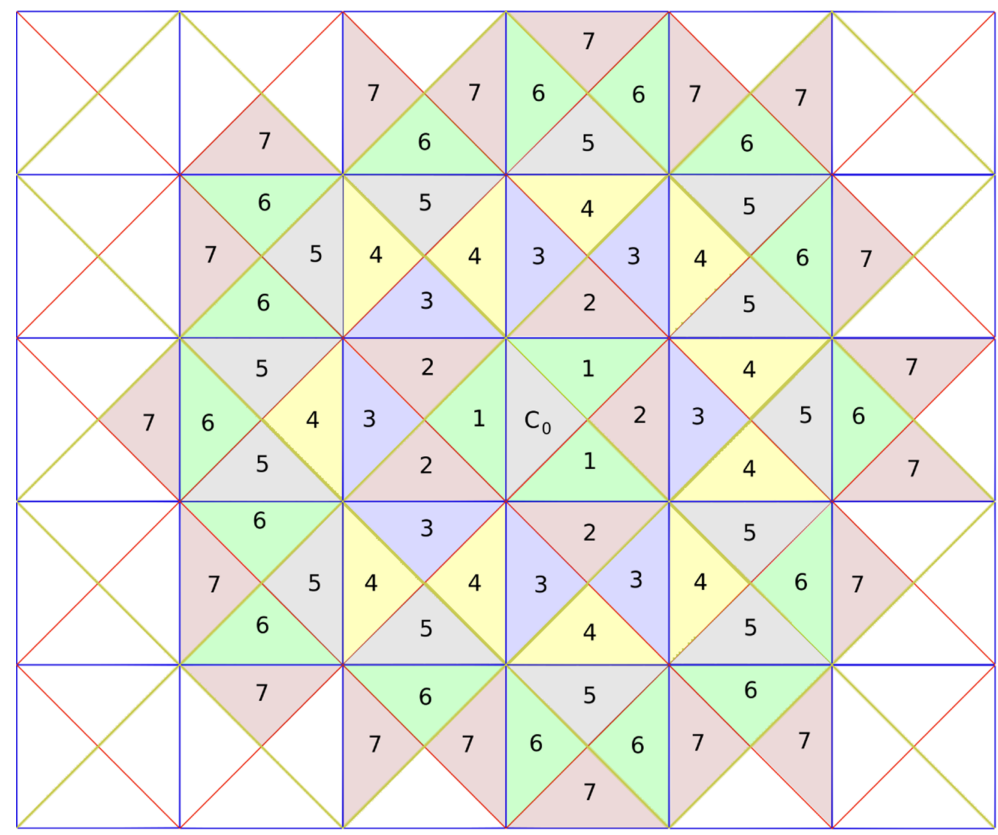

FiguRE 1. Illustration of the height of chambers in an apartment when $\mathcal{G}$ is of type $\mathrm{C}_{2}$.

If $\psi$ is an affine root, we set the associated affine hyperplane as :

$$
H_{\psi}:=\text { the zero locus (an affine hyperplane) of } \psi \text {. }
$$

We also use the notation $H_{ \pm \psi}$ for this affine hyperplane.

For any facet $F$ (not necessarily a face) of $D$, we set

$$
\Psi(F):=\text { set of affine roots } \psi \text { which vanishes on } F \text {. }
$$


A face $F$ (facet of dimension $(\ell-1)$ ) of $D$ is in the zero hyperplane set of a unique pair of affine roots $\pm \psi$, i.e., $\Psi(F)=\{ \pm \psi\}$. When $F$ is a face of $D$, set

$$
\begin{aligned}
r_{F}\left(\in W^{\mathrm{a}}\right) & =\text { the affine reflection across the hyperplane } H_{ \pm \psi}, \\
\operatorname{opp}_{F}(D) & =\text { the chamber obtained from } D \text { by reflection across } F .
\end{aligned}
$$

Any chamber $D$ of $\mathcal{A}$ can be obtained from $C_{0}$ by a composition of reflections $r_{F}\left(\in W^{\mathrm{a}}\right)$. We find it useful to define for a pair of roots $\{ \pm \gamma\} \subset \Phi$, the $\{ \pm \gamma\}$ height of a chamber $D$ with respect to $C_{0}$ as:

$$
\operatorname{ht}_{C_{0}}^{ \pm \gamma}(D):=\left\{\begin{array}{l}
\text { The number of affine hyperplanes } H_{\psi} \text { satisfying: } \\
\text { (i) } \operatorname{grad}(\psi)= \pm \gamma, \text { and } \\
\text { (ii) } H_{\psi} \text { separates } C_{0} \text { and } D .
\end{array}\right.
$$

Then we have the following.

Lemma 2.3.5. Fix a base chamber $C_{0}$ in $\mathcal{A}$. If $D$ is a chamber in $\mathcal{A}$, then the minimum number of affine reflections needed to take $C_{0}$ to $D$ is the sum

$$
\mathrm{ht}_{C_{0}}(D)=\sum_{\{ \pm \gamma\}} \mathrm{ht}_{C_{0}}^{ \pm \gamma}(D)
$$

over all pairs of roots in $\Phi$.

Proof. In $W^{\text {a }}$, let $X=\mathcal{S} / \mathcal{S}_{\mathrm{c}}$, and $W=N_{\mathcal{G}}(\mathcal{S}) / \mathcal{S}$, the finite Weyl group of $\mathcal{G}$. If we take a hyperspecial point $x_{0}$ in $\mathcal{A}$, then every element $w \in W$, has a representative $n_{w} \in\left(\mathcal{G}_{x_{0}, 0} \cap N_{\mathcal{G}}(\mathcal{S})\right)$, which is unique modulo $\mathcal{S}_{c}$. Let $W_{x_{0}}=\left\{n_{w} \mid w \in W\right\}$ be a set of such representatives of $W$. Then, any $n \in N_{\mathcal{G}}(\mathcal{S})$ can be written as $n=x n_{w}$ with $x \in \mathcal{S}$ and $n_{w} \in W_{x_{0}}$.

Suppose $D$ is a chamber of $\mathcal{A}$. Take $n=x n_{w} \in N_{\mathcal{G}}(\mathcal{S})$ so that $D=\left(x n_{w}\right) C_{0}$. Fix a positive system of roots $\Phi^{+} \subset \Phi$. Denote the negative roots as $\Phi^{-}$. By Proposition 1.23 of [M], the Bruhat length $\operatorname{Bru}(n)$ of $n$ is

$$
\operatorname{Bru}(n)=\operatorname{Bru}\left(x n_{w}\right)=\sum_{\substack{\alpha \in \Phi^{+} \\ w^{-1}(\alpha) \in \Phi^{+}}}|\langle x, \alpha\rangle|+\sum_{\substack{\alpha \in \Phi^{+} \\ w^{-1}(\alpha) \in \Phi^{-}}}|\langle x, \alpha\rangle-1| .
$$

For $\alpha \in \Phi^{+}$, the geometric meaning of the function

$$
\operatorname{Ht}^{\alpha}\left(x n_{w}\right):= \begin{cases}|\langle x, \alpha\rangle| & \text { when } w^{-1}(\alpha) \in \Phi^{+} \\ |\langle x, \alpha\rangle-1| & \text { when } w^{-1}(\alpha) \in \Phi^{-}\end{cases}
$$

is precisely the function $\mathrm{ht}_{C_{0}}^{ \pm \alpha}$. The lemma follows.

We note that if $\mathcal{A}^{\prime}$ is another apartment of $\mathcal{B}$ containing $C_{0}$, then the two height functions agree on chambers in the intersection $\mathcal{A} \cap \mathcal{A}^{\prime}$. Thus, there is a unique extension of the height function $\mathrm{ht}_{C_{0}}$ to all the chambers of $\mathcal{B}$.

We observe that if $F$ is a face of a chamber $D \subset \mathcal{A}$, and $\Psi(F)=\{ \pm \psi\}$, then for $z \in \operatorname{recess}(F)$ and small positive $\epsilon$, the point $z+\epsilon \operatorname{grad}(\psi)$ is either in $D$ or $\operatorname{opp}_{F}(D)$. With respect to $D$, we define:

(i) $\psi$ is outwards oriented if $z+\epsilon \operatorname{grad}(\psi)$ is in $\operatorname{opp}_{F}(D)$ for small positive $\epsilon$,

(ii) $\psi$ is inwards oriented if $z+\epsilon \operatorname{grad}(\psi)$ is in $D$ for small positive $\epsilon$. 
Suppose $D$ is a chamber in the apartment $\mathcal{A}$. Set

$$
\begin{aligned}
& c(D)=\left\{F \text { a face of } D-\mathrm{ht}_{C_{0}}\left(\operatorname{opp}_{F}(D)\right)=\mathrm{ht}_{C_{0}}(D)+1\right\}, \\
& p(D)=\left\{F \text { a face of } D-\mathrm{ht}_{C_{0}}\left(\operatorname{opp}_{F}(D)\right)=\mathrm{ht}_{C_{0}}(D)-1\right\} .
\end{aligned}
$$

Clearly any face of $D$ belongs to either $c(D)$ or $p(D)$. Mnemonically, the set $p(D)$ (resp., $c(D))$ is the set of "parent" or "inward" (resp., "child" or "outward") faces of the chamber $D$.

Proposition 2.3.8. Suppose $D$ is a chamber of an apartment $\mathcal{A}$, and $F$ is a face of $D$, and $\mathrm{ht}_{C_{0}}\left(\operatorname{opp}_{F}(D)\right)=\mathrm{ht}_{C_{0}}(D)+1$. Write $\Psi(F)=\{ \pm \psi\}$ (notation (2.3.2)), and choose $\psi$ to be outward oriented for $D$ (notation 2.3.6). Set $\alpha=\operatorname{grad}(\psi)$. Then for any $y \in \operatorname{recess}(D)$ and $x \in \operatorname{recess}(F)$,

$$
\mathcal{G}_{y, 0+} \cap \mathcal{U}_{\alpha}=\mathcal{G}_{x, 0} \cap \mathcal{U}_{\alpha} .
$$

Proof. One verifies both subgroups equal the affine root subgroup $x_{\psi+1}$.

2.4. Chamber based sectors in an apartment. We fix a maximal torus $\mathcal{S}$ and its associated apartment $\mathcal{A}=\mathcal{A}(\mathcal{S})$. If $\psi$ is an affine root, set

$$
H_{\psi>0}:=\{x \in \mathcal{A} \mid \psi(x)>0\} \quad \text { and } \quad H_{\psi \geq 0}:=\{x \in \mathcal{A} \mid \psi(x) \geq 0\} .
$$

Suppose $\Phi^{+}$is a choice of positive roots in $\Phi=\Phi(\mathcal{S})$, and $C_{0}$ is a chamber. Set:

$$
S\left(C_{0}, \Phi^{+}\right):=\bigcap_{\substack{\operatorname{grad}(\psi) \in \Phi^{+} \\ C_{0} \subset H_{\psi \geq 0}}} H_{\psi \geq 0} .
$$

We call such a set the $C_{0}$-chamber based sector with respect to the positive roots $\Phi^{+}$. Figure 2 illustrates $3 C_{0}$-based sectors in $C_{2}$.

Proposition 2.4.3. The chamber based sector $S\left(C_{0}, \Phi^{+}\right)$is the set of chambers which can be obtained from $C_{0}$ by repeated application of affine reflections $s_{\psi}$ with $\operatorname{grad}(\psi) \in \Phi^{+}$, and outwards oriented.

We note:

- The union of the sets $S\left(C_{0}, \Phi^{+}\right)$, as $\Phi^{+}$runs over positive roots subsets of $\Phi$, is $\mathcal{A}$.

- A chamber $D$ can belong to more than one $S\left(C_{0}, \Phi^{+}\right)$. This happens when $D$ is near a (Weyl chamber) wall.

If $D$ is a chamber of $\mathcal{A}$, set

$$
\begin{aligned}
& \mathcal{R}\left(C_{0}, D\right):=\left\{\Phi^{+} \text {a set of positive roots } \mid D \subset S\left(C_{0}, \Phi^{+}\right)\right\}, \\
& B\left(C_{0}, D\right):=\bigcap_{\Phi^{+} \in \mathcal{R}\left(C_{0}, D\right)} S\left(C_{0}, \Phi^{+}\right) .
\end{aligned}
$$

Alternatively, we define a subset $\Phi\left(C_{0}, D\right)$ of the roots $\Phi$ as follows:

(i) Suppose $\alpha$ is a root and there is an affine root $\psi$ with $\operatorname{grad}(\psi)=\alpha$, and the zero hyperplane $H_{\psi}\left(=H_{-\psi}\right)$ separates $C_{0}$ and $D$. We define $D$ to be $\operatorname{grad}(\psi)$ oriented with respect to $C_{0}$ if for $y \in H_{\psi}$ and $x \in \operatorname{recess}(D)$ we have:

$$
\langle(x-y), \operatorname{grad}(\psi)\rangle>0 \text {. }
$$




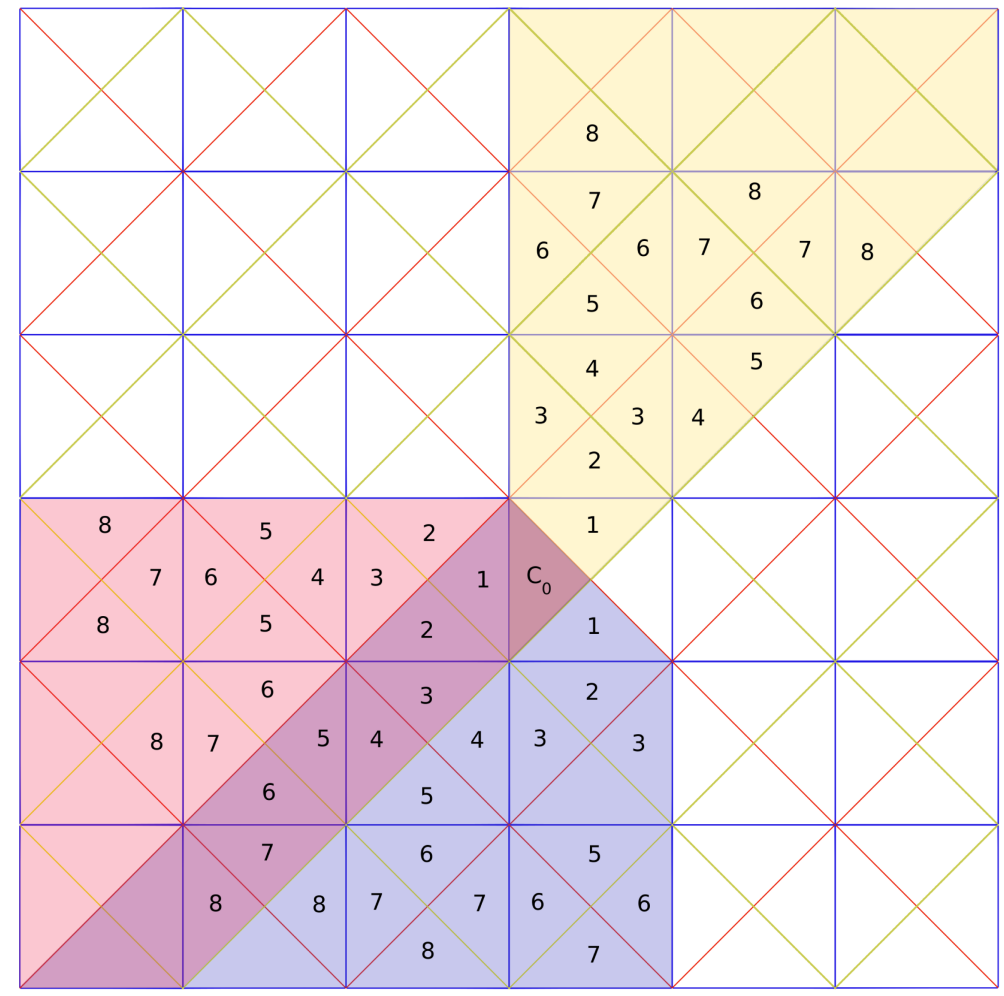

FIGURE 2. Illustration of three chamber based sectors when $\mathcal{G}$ is of type $\mathrm{C}_{2}$.

Obviously, $D$ is either $\operatorname{grad}(\psi)$ or $\operatorname{grad}(-\psi)$ oriented with respect to $C_{0}$. Also, $D$ is $\operatorname{grad}(\psi)$ oriented if, for any positive $\epsilon$, the point $y+\epsilon \operatorname{grad}(\psi)$ and points of recess $(D)$ are on the same side of the zero hyperplane $H_{\psi}$. Set $\alpha=\operatorname{grad}(\psi)$. Clearly, there is an affine root $\alpha-k$ with smallest possible $k$ so that $H_{\alpha-(k+1)}$ separates $C_{0}$ and $D$, but $H_{\alpha-k}$ does not, i.e.,

$$
H_{(\alpha-k),>0}:=\{x \in \mathcal{A} \mid(\alpha-k)(x)>0\}
$$

contains recess $\left(C_{0}\right)$ and recess $(D)$, and is the smallest of the affine root half-spaces $H_{\psi,>0}$ with $\operatorname{grad}(\psi)=\alpha$ which does so.

(ii) Suppose $\alpha$ is a root so that for any affine root $\psi$ with $\operatorname{grad}(\psi)= \pm \alpha$, the zero hyperplane $H_{\psi}$ of $\psi$ does not separate $C_{0}$ and $D$. Here, by replacing $\alpha$ by $-\alpha$ if necessary, there is an affine root $\psi=\alpha-k$ so that $\operatorname{recess}\left(C_{0}\right)$ and $\operatorname{recess}(D)$ lie in the band

$$
B_{\alpha}:=\{x \in \mathcal{A} \mid k<\alpha(x)<(k+1)\} .
$$

We define a canonical set $\Phi\left(C_{0}, D\right)$ as:

$$
\Phi\left(C_{0}, D\right)=\text { the union of the roots } \operatorname{grad}(\psi) \text { in (i) . }
$$

In the situation when there are no roots of type (ii), then the roots $\Phi\left(C_{0}, D\right)$ are a positive system of roots, and $B\left(C_{0}, D\right)=S\left(C_{0}, \Phi\left(C_{0}, D\right)\right)$. 
We explain the significance of the roots of types (i) and (ii). Suppose $x \in C_{0}$ and $y \in D$. Then, as we move from $x$ to $y$ along the line segment $(1-t) x+t y$ :

- For $\alpha \in \Phi\left(C_{0}, S\right)$,

$$
\begin{aligned}
\mathcal{G}_{((1-t) x+t y), 0^{+}} \cap \mathcal{U}_{\alpha} & \text { increases from } \mathcal{G}_{x, 0^{+}} \cap \mathcal{U}_{\alpha} \text { to } \mathcal{G}_{y, 0^{+}} \cap \mathcal{U}_{\alpha}, \\
\mathcal{G}_{((1-t) x+t y), 0^{+}} \cap \mathcal{U}_{-\alpha} & \text { decreases from } \mathcal{G}_{x, 0^{+}} \cap \mathcal{U}_{-\alpha} \text { to } \mathcal{G}_{y, 0^{+}} \cap \mathcal{U}_{-\alpha} .
\end{aligned}
$$

- If $\pm \alpha$ is a root of type (ii), then

$$
\mathcal{G}_{((1-t) x+t y), 0^{+}} \cap \mathcal{U}_{\alpha} \text { is constant . }
$$

The roots of type (ii) are the roots of a Levi subgroup $\mathcal{L}$ containing $\mathcal{S}$. The roots of type (i) are those of a unipotent radical $\mathcal{V}$ of a parabolic subgroup $\mathcal{P}=\mathcal{L} \mathcal{V}$. Let $\overline{\mathcal{V}}$ denote the opposite radical $\mathcal{V}$. For $x \in \mathcal{A}(\mathcal{S})$, set

$$
\mathcal{L}_{x, 0^{+}}:=\mathcal{G}_{x, 0^{+}} \cap \mathcal{L}, \quad \mathcal{V}_{x, 0^{+}}:=\mathcal{G}_{x, 0^{+}} \cap \mathcal{V}, \quad \overline{\mathcal{V}}_{x, 0^{+}}:=\mathcal{G}_{x, 0^{+}} \cap \overline{\mathcal{V}} .
$$

Then

$$
\mathcal{G}_{x, 0^{+}}=\mathcal{L}_{x, 0^{+}} \mathcal{V}_{x, 0^{+}} \overline{\mathcal{V}}_{x, 0^{+}} \quad \text { in any order } .
$$

Proposition 2.4.7. Suppose $D$ is a chamber of $\mathcal{A}$. Define the Levi $\mathcal{L}$ and $\mathcal{V}$ as above. Let $c(D)$ be the set of child faces of $D$, and suppose $E, F \in \mathcal{C}(c(D))$, so that $F$ is a face of $E$. Then, for $y \in \operatorname{recess}(E)$, and $x \in \operatorname{recess}(F)$ :

$$
\mathcal{V}_{y, 0^{+}}=\mathcal{V}_{x, 0^{+}} \text {and } \quad \mathcal{L}_{y, 0^{+}}=\mathcal{L}_{x, 0^{+}} .
$$

Proof. Apply Proposition 2.3.8.

2.5. A finiteness condition on chambers. Define a non-empty set of affine root pairs $\mathcal{X}=\left\{ \pm \psi_{1}, \ldots, \pm \psi_{k}\right\}$ to be permissible if :

- $\operatorname{grad}\left(\psi_{1}\right), \ldots, \operatorname{grad}\left(\psi_{k}\right)$ are linearly independent. Here, we have selected one affine root from each pair. Let

$$
\mathcal{A S}(\mathcal{X}):=H_{ \pm \psi_{1}} \cap \cdots \cap H_{ \pm \psi_{k}}
$$

be an $(\ell-k)$-dimensional affine subspace of $\mathcal{A}$.

- There exists a chamber $D$ so that each intersection $H_{\psi_{i}} \cap D$ is a face of $D$. We name this relationship as the chamber $D$ being incident with $\mathcal{X}$. In this situation, the intersection $D \cap \mathcal{A S}(\mathcal{X})$ is an $(\ell-k)$-dimensional facet of $D$.

If $D$ is a chamber, set

(2.5.2) $\Xi(D):=$ set of affine root pairs $\pm \psi$ so that $H_{ \pm \psi} \cap D$ is a face of $D$.

We observe :

- If $D$ is a chamber, then any non-empty proper subset $\mathcal{X}=\left\{ \pm \psi_{i_{1}}, \ldots, \pm \psi_{i_{k}}\right\}$

- of $\Xi(D)$ is permissible.

When $D$ is incident with (a permissible set) $\mathcal{X}$, let

$$
\mathcal{X}_{D}^{\prime}=\left\{ \pm \phi_{k+1}, \ldots, \pm \phi_{\ell+1}\right\}=\Xi(D) \backslash \mathcal{X}
$$

be the affine root pairs so that the remaining $(\ell+1-k)$ faces of $D$ lie in the affine hyperplanes $H_{ \pm \phi_{k+1}}, \ldots, H_{ \pm \phi_{\ell+1}}$. The affine subspace $\mathcal{A S}\left(\mathcal{X}_{D}^{\prime}\right)$ has dimension $(k-$ 1 ), and the two subsimplices $(D \cap \mathcal{A S}(\mathcal{X}))$ and $\left(D \cap \mathcal{A S}\left(\mathcal{X}_{D}^{\prime}\right)\right)$ of the simplex $D$ 
are opposite subsimplices, i.e., any vertex of $D$ lies in precisely one of the two subsimplices. Define vector spaces

$$
\begin{aligned}
& \operatorname{Vect}(\mathcal{A S}(\mathcal{X})):=\text { translations of } \mathcal{A} \text { which leave } \mathcal{A S}(\mathcal{X}) \text { invariant, } \\
& \operatorname{Vect}\left(\mathcal{A S}\left(\mathcal{X}_{D}^{\prime}\right)\right):=\text { translations of } \mathcal{A} \text { which leave } \mathcal{A S}\left(\mathcal{X}_{D}^{\prime}\right) \text { invariant. }
\end{aligned}
$$

A consequence of the oppositeness of the two subsimplices $(D \cap \mathcal{A S}(\mathcal{X}))$ and $(D \cap$ $\left.\mathcal{A S}\left(\mathcal{X}_{D}^{\prime}\right)\right)$ of $D$ is that $\operatorname{Vect}(\mathcal{A S}(\mathcal{X}))$ and $\operatorname{Vect}\left(\mathcal{A S}\left(\mathcal{X}_{D}^{\prime}\right)\right)$ are linearly independent. They span a subspace of dimension $(\ell-1)$.

We note if $\mathcal{X}$ is permissible and $\operatorname{dim}(\mathcal{A S}(\mathcal{X}))=(\ell-k)>0$, then there are infinitely many distinct chambers $D$ incident with $\mathcal{X}$, and $\mathcal{A S}(\mathcal{X})$ is tiled by the intersections $D \cap \mathcal{A S}(\mathcal{X})$ as $D$ runs over all chambers incident with $\mathcal{X}$. Indeed, let $\mathcal{S}_{c}$ be the maximal bounded (hence compact) subgroup of $\mathcal{S}$. We recall the discrete (free commutative of $\operatorname{rank} \ell$ ) group $\Gamma(\mathcal{A}):=\mathcal{S} / \mathcal{S}_{c}$ acts as translations on $\mathcal{A}$. Set

$$
\begin{aligned}
\Gamma(\mathcal{A S}(\mathcal{X})): & =\text { subgroup of } \Gamma(\mathcal{A}) \text { preserving } \mathcal{A S}(\mathcal{X}) \\
\Gamma\left(\mathcal{A S}\left(\mathcal{X}_{D}^{\prime}\right)\right): & =\text { subgroup of } \Gamma(\mathcal{A}) \text { preserving } \mathcal{A S}\left(\mathcal{X}_{D}^{\prime}\right) .
\end{aligned}
$$

These subgroups are of ranks $(\ell-k)$ and $(k-1)$, respectively, and

$$
\operatorname{Vect}(\mathcal{A S}(\mathcal{X}))=\Gamma(\mathcal{A S}(\mathcal{X})) \otimes_{\mathbb{Z}} \mathbb{R}, \quad \operatorname{Vect}\left(\mathcal{A S}\left(\mathcal{X}_{D}^{\prime}\right)\right)=\Gamma\left(\mathcal{A S}\left(\mathcal{X}_{D}^{\prime}\right)\right) \otimes_{\mathbb{Z}} \mathbb{R} .
$$

If $D$ is an incident chamber to $\mathcal{X}$, and $x \in \Gamma(\mathcal{A S}(\mathcal{X}))$, then the translated chamber $D+x$ is also incident. We deduce there are finitely many distinct $\mathcal{X}$ incident chambers $D_{1}, \ldots, D_{M}$ so that the union of facets

$$
\mathrm{UF}=\left(D_{1} \cap \mathcal{A S}(\mathcal{X})\right) \cup \ldots \cup\left(D_{M} \cap \mathcal{A S}(\mathcal{X})\right)
$$

is a fundamental domain for the translation action of $\Gamma(\mathcal{A S}(\mathcal{X}))$ on $\mathcal{A S}(\mathcal{X})$.

Proposition 2.5.5. Fix a chamber $C_{0}$, and suppose $\mathcal{X}=\left\{ \pm \psi_{1}, \ldots, \pm \psi_{k}\right\}$ is a permissible set of affine roots. Then, the number of chambers $D$ which are incident with $\mathcal{X}$, and satisfy

$$
c(D)=\left\{H_{ \pm \psi_{i}} \cap D \mid 1 \leq i \leq k\right\}
$$

(notation (2.3.7)) is finite.

We remark about the extreme cases:

- If $k=\ell$, and $\mathcal{X}$ is permissible, then there is a unique chamber incident with $\mathcal{X}$, so the assertion is obvious.

- If $k=1$, the singleton pair set $\mathcal{X}=\{ \pm \psi\}$ is automatically permissible, $\mathcal{A S}(\mathcal{X})$ is $H_{ \pm \psi}$, and for any $\mathcal{X}$-incident chamber $D$, the intersection $E=$ $(D \cap \mathcal{A S}(\mathcal{X}))$ is a face of $D$, and $\left(D \cap \mathcal{A S}\left(\mathcal{X}_{D}^{\prime}\right)\right)$ is the vertex opposite to $E$.

Proof. Suppose $D$ is a chamber incident with $\mathcal{X}$, and $\mathcal{X}_{D}^{\prime}=\Xi(D) \backslash \mathcal{X}$. For each affine root pair $\pm \psi_{i}$ in $\mathcal{X}$, or $\pm \phi_{j}$ in $\mathcal{X}_{D}^{\prime}$ it is convenient for us to select a preferred affine root. We do this by designating $\psi_{i}$ to be the affine root so that the chamber $D$ lies in the half-space $H_{\psi_{i} \leq 0}$ for $1 \leq i \leq k$. Similarly, for a pair $\pm \phi_{j}$ in $\mathcal{X}_{D}^{\prime}$, we designate $\phi_{j}$ to be the affine root so that $D$ is in the half-space $H_{\phi_{i} \geq 0}$.

The proposition's hypothesis that $c(D)$ is the set $\left\{H_{\psi_{i}} \cap D \mid 1 \leq i \leq k\right\}$ means

$$
C_{0} \subset \operatorname{Cone}(\mathcal{X}, D):=\left(\bigcap_{1 \leq i \leq k} H_{\psi_{i} \leq 0}\right) \bigcap\left(\bigcap_{(k+1) \leq i \leq(\ell+1)} H_{\phi_{i} \leq 0}\right) .
$$


We define and observe:

$$
\begin{aligned}
& \text { Cone } \mathcal{X}:=\left(\bigcap_{1 \leq i \leq k} H_{\psi_{i} \leq 0}\right) \quad \begin{array}{l}
\text { depends only on the permissible set } \mathcal{X} \text {, and } \\
\text { not on the chamber } D \text {, }
\end{array} \\
& \text { Cone }_{D}:=\left(\bigcap_{(k+1) \leq i \leq(\ell+1)} H_{\phi_{i} \leq 0}\right) \text { is dependent on the incident chamber } D \text {. }
\end{aligned}
$$

Obviously, cones Cone $\mathcal{X}$ and $\operatorname{Cone}_{D}$ are $\operatorname{Vect}(\mathcal{A S}(\mathcal{X}))$-invariant and $\operatorname{Vect}\left(\mathcal{A S}\left(\mathcal{X}_{D}^{\prime}\right)\right)$ invariant, respectively. If we translate the chamber $D$ by $y \in \Gamma(\mathcal{A S}(\mathcal{X}))$, to obtain another incident chamber $D+y$, we have :

$$
\text { Cone }_{D+y}=\left(\text { Cone }_{D}\right)+y \text { and } \operatorname{Cone}(\mathcal{X}, D+y)=\operatorname{Cone}(\mathcal{X}, D)+y \text {. }
$$

We consider the $\left(\operatorname{Vect}(\mathcal{A S}(\mathcal{X}))+\operatorname{Vect}\left(\mathcal{A S}\left(\mathcal{X}_{D}^{\prime}\right)\right)\right.$-invariant band

$$
\begin{aligned}
\mathcal{B} n\left(C_{0}\right): & =C_{0}+\operatorname{Vect}(\mathcal{A S}(\mathcal{X}))+\operatorname{Vect}\left(\mathcal{A S}\left(\mathcal{X}_{D}^{\prime}\right)\right) \\
& =\left\{x+y+z \mid x \in C_{0}, y \in \operatorname{Vect}(\mathcal{A S}(\mathcal{X})), z \in \operatorname{Vect}\left(\mathcal{A S}\left(\mathcal{X}_{D}^{\prime}\right)\right)\right\} .
\end{aligned}
$$

If $C_{0}$ lies in both $\operatorname{Cone}(\mathcal{X}, D)$ and $\operatorname{Cone}(\mathcal{X}, D+y)$, we must have

$$
C_{0} \subset\left(\operatorname{Cone}(\mathcal{X}, D) \cap \mathcal{B} n\left(C_{0}\right)\right) \cap\left(\left(\operatorname{Cone}(\mathcal{X}, D) \cap \mathcal{B} n\left(C_{0}\right)\right)+y\right) .
$$

Since the set $\left(\right.$ Cone $\left.(\mathcal{X}, D) \cap \mathcal{B} n\left(C_{0}\right)\right)$ is compact, and $\Gamma(\mathcal{A S}(\mathcal{X}))$ are (discrete) translations, the set of $y \in \Gamma(\mathcal{A S}(\mathcal{X}))$ satisfying (2.5.7) is finite.

To summarize: If $D$ is a chamber incident to (a permissible set) $\mathcal{X}$ and $C_{0} \subset$ $\operatorname{Cone}(\mathcal{X}, D)$, then there are only finitely many $y \in \Gamma(\mathcal{A S}(\mathcal{X}))$ satisfying $C_{0} \subset$ Cone $(\mathcal{X}, D+y)$ as well. As mentioned above, we can find finitely many disjoint incident chambers so that the union (2.5.4) of their closures is a fundamental domain for $\Gamma(\mathcal{A S}(\mathcal{X}))$. The statement of the proposition follows.

2.6. A criterion for a distribution to be essentially compact. A much exploited criterion (see [BD, §1.7]) for a distribution $D$ to be essentially compact is the following.

Lemma 2.6.1. Suppose $D$ is a $\mathcal{G}$-invariant distribution on $\mathcal{G}$. Then, $D$ is essentially compact, and therefore in the Bernstein center $\mathcal{Z}(\mathcal{G})$, if and only if for any open compact subgroup $J$, the function $D \star 1_{J}$ is compactly supported.

\section{Harish-Chandra CUSPIDAl Classes AND idempotents}

Throughout this section, $G$ is a connected reductive linear algebraic group defined over a finite field $\mathbb{F}_{q}$. When $P$ is a parabolic subgroup of $G$, we denote its unipotent radical by $\operatorname{rad}(P)$. Given a fixed Borel subgroup $B \subset G$, a $B$-standard parabolic subgroup, is one which contains $\mathrm{B}$. Let $\mathcal{G}=\mathrm{G}\left(\mathbb{F}_{q}\right), \mathcal{B}=\mathrm{B}\left(\mathbb{F}_{q}\right), \mathcal{P}=\mathrm{P}\left(\mathbb{F}_{q}\right)$, etc., denote the groups of $\mathbb{F}_{q}$-rational points. We take the Haar measure on $\mathcal{G}$ and its subgroups to be the point mass.

3.1. A convolution property of idempotents of $\operatorname{rad}(\mathcal{P})$. For $\mathcal{H}$ any subgroup of $\mathcal{G}$, and a representation $\kappa$ of $\mathcal{H}$, let $\Theta_{\kappa}$ signify its character. The function

$$
e_{\kappa}:=\frac{1}{\#(\mathcal{H})} \operatorname{deg}(\kappa) \Theta_{\kappa}=\frac{1}{\#(\mathcal{H})} \operatorname{deg}(\kappa) \sum_{g \in \mathcal{H}} \Theta_{\kappa}(g) \delta_{g}
$$

is a convolution idempotent (in $C(\mathcal{G})$ ).

Take $\mathcal{T}$ to be a maximal torus in $\mathcal{B}$, let $\Phi^{+}=\Phi^{+}(\mathcal{T})$ be the positive roots determined by $\mathcal{B}$, and let $\Delta$ be the simple roots of $\Phi^{+}$. The $\mathcal{B}$-standard parabolic 
subgroups are indexed by subsets of $\Delta$. A subset to $\subset \Delta$ corresponds to the parabolic subgroup $\mathcal{P}_{\text {do }}$ generated by the Borel subgroup $\mathcal{B}$ and the root groups $\mathcal{U}_{-\alpha}, \alpha \in$ ot. In particular, $\mathcal{P}_{\emptyset}=\mathcal{B}$, and $\mathcal{P}_{\Delta}=\mathcal{G}$.

Lemma 3.1.2. Suppose $\mathcal{R} \supsetneq \mathcal{Q}$ are two $\mathcal{B}$-standard parabolic subgroups and $\mathcal{V}=$ $\operatorname{rad}(\mathcal{Q})$ are their unipotent radicals. Then,

$$
\left(\sum_{\mathcal{R} \supset \mathcal{P} \supset \mathcal{B}}(-1)^{\operatorname{rank}(\mathcal{P})} e_{\operatorname{rad}(\mathcal{P})}\right) \star e_{\mathcal{V}}=\text { zero function }
$$

Proof. As mentioned above, we index a $\mathcal{B}$-standard parabolic subgroup by subset do $\subset \Delta$. For convenience, we use the notation $e_{d t}$ to denote the idempotent $e_{\operatorname{rad}\left(P_{d 0}\right)}$. Then, $e_{\text {do }} \star e_{\beta}=e_{\text {don } \beta}$. Denote by $Q$ and $\mathcal{R}$ the subsets of $\Delta$ satisfying $\mathcal{Q}=\mathcal{P}_{Q}$, and $\mathcal{R}=\mathcal{P}_{\mathscr{R}}$.

The alternating sum of the idempotents is a sum over $2^{\#(\mathcal{R})}$ terms. Since $\mathcal{Q} \subsetneq \mathcal{R}$, we have $Q \subsetneq \mathcal{R}$, i.e., the complement $Q^{\prime}:=R \backslash Q$ is non-empty. For each subset $q \subset Q$, we consider the subsets of $\mathcal{R}$ obtained from $q$ by adding a subset $q^{\prime}$ of $Q^{\prime}$. The convolution of $e_{Q}$ with the sum over these $2^{\#\left(Q^{\prime}\right)}$ subsets is, up to \pm 1 :

$$
\begin{aligned}
\left(\sum_{q^{\prime} \subset Q^{\prime}}(-1)^{\#\left(q \cup q^{\prime}\right)} e_{q \cup q^{\prime}}\right) \star e_{Q} & =(-1)^{\#(q)} \sum_{q^{\prime} \subset Q^{\prime}}(-1)^{\#\left(q^{\prime}\right)} e_{q \cup q^{\prime}} \star e_{\mathrm{Q}} \\
& =(-1)^{\#(q)} \sum_{q^{\prime} \subset Q^{\prime}}(-1)^{\#\left(q^{\prime}\right)} e_{q} \\
& =\text { zero function } .
\end{aligned}
$$

The statement of the lemma follows.

3.2. Harish-Chandra cuspidal classes. Define a Harish-Chandra cuspidal class to be an equivalence class $\mathscr{L}$ of pairs $(\mathcal{L}, \sigma)$, modulo conjugation by $\mathcal{G}$, consisting of a Levi subgroup $\mathcal{L} \subset \mathcal{G}$ and an irreducible cuspidal representation $\sigma$ of $\mathcal{L}$. So, $(\mathcal{L}, \sigma) \sim\left(\mathcal{L}^{\prime}, \sigma^{\prime}\right)$, if there exists $g \in G$ satisfying $\mathcal{L}=g^{-1} \mathcal{L}^{\prime} g$, and $\sigma(x)=\sigma^{\prime}\left(g x g^{-1}\right)$ for all $x \in \mathcal{L}$. We recall two parabolic subgroups $\mathcal{P}$ and $\mathcal{P}^{\prime}$ are associate if they have Levi factors $\mathcal{L}$ and $\mathcal{L}^{\prime}$ which are $\mathcal{G}$ conjugate.

A Harish-Chandra cuspidal class $\mathscr{L}$ defines up to conjugation by $\mathcal{G}$ a Levi subgroup $\mathcal{L}$, and thus an associativity class of parabolic subgroups, and possibly several irreducible cuspidal representations of $\mathcal{L}$. A Harish-Chandra cuspidal class $\mathcal{L}$ determines a subcategory $\mathcal{R}_{\mathscr{L}}$ of the category of representations $\mathcal{R}$ of $\mathcal{G}$, namely

$$
\mathcal{R}_{\mathscr{L}}=\left\{\begin{array}{l}
\text { Representations of } \mathcal{G} \text { whose irreducible subrepresentations are } \\
\text { equivalent to representations in the induced representation } \\
\operatorname{Ind}_{\mathcal{L} \mathcal{U}}(\sigma), \text { where }(\mathcal{L}, \sigma) \in \mathcal{L}, \text { and } \mathcal{L U} \text { is a parabolic subgroup con- } \\
\text { taining the Levi subgroup } \mathcal{L} .
\end{array}\right.
$$

If $\mathscr{L}$ is a Harish-Chandra cuspidal class, take $(\mathcal{L}, \sigma) \in \mathscr{L}$, and set

$$
e_{\mathcal{L}, \mathscr{L}}:=\frac{1}{\#(\mathcal{L})} \sum_{\substack{\kappa \in \widehat{\mathcal{L}} \\(\mathcal{L}, \kappa) \in \mathscr{L}}} \operatorname{deg}(\kappa) \Theta_{\kappa} .
$$


For a parabolic subgroup $\mathcal{P}=\mathcal{L U}$, set

$$
e_{\mathcal{P}, \mathscr{L}}:=\frac{1}{\#(\mathcal{U})} \text { inflation to } \mathcal{P} \text { of } e_{\mathcal{L}, \mathscr{L}},
$$

We extend $e_{\mathcal{L}, \mathscr{L}}, e_{\mathcal{P}, \mathscr{L}}$, and $e_{\mathcal{U}}$ to functions on $\mathcal{G}$ by setting their values outside $\mathcal{L}$, $\mathcal{P}$, and $\mathcal{U}$, respectively, to be zero. In $\mathcal{G}$, it is obvious

$$
e_{\mathcal{P}, \mathscr{L}}=e_{\mathcal{L}, \mathscr{L}} \star e_{\mathcal{U}}
$$

To any parabolic subgroup $\mathcal{Q}$, we wish to attach an idempotent $e_{Q, \mathscr{L}}$. We do this as follows: Fix $(\mathcal{L}, \kappa) \in \mathscr{L}$. Let $\mathcal{Q}=\mathcal{M V}$ be a Levi decomposition of $\mathcal{Q}$. We consider whether or not there is a conjugate of $\mathcal{L}$ contained in $\mathcal{M}$.

- When a conjugate $\mathcal{L}^{\prime}=g \mathcal{L} g^{-1}$ of $\mathcal{L}$ is contained in $\mathcal{M}$, we take $\mathcal{P}^{\prime}=\mathcal{L}^{\prime} \mathcal{U}^{\prime}$ to be a parabolic subgroup (of $\mathcal{G}$ ) which is contained in $\mathcal{Q}$. Then $\mathcal{L}^{\prime}\left(\mathcal{M} \cap \mathcal{U}^{\prime}\right)$ is a parabolic subgroup of $\mathcal{M}$. Set

$$
e_{\mathcal{M}, \mathscr{L}}:=\frac{1}{\#(\mathcal{M})} \sum_{\tau \in \widehat{\mathcal{M}}}^{\prime} \operatorname{deg}(\tau) \Theta_{\tau}
$$

where the sum $\sum^{\prime}$ is over the irreducible representations $\tau$ of $\mathcal{M}$ for which the invariants $\tau^{\mathcal{M} \cap \mathcal{U}^{\prime}}$ contain an irreducible representation $\sigma^{\prime}$ of $\mathcal{L}^{\prime}$ with $\left(\mathcal{L}^{\prime}, \sigma^{\prime}\right) \in \mathscr{L}$. We also set

$\mathcal{R}_{\mathcal{M}, \mathscr{L}}=\left\{\begin{array}{l}\text { the subcategory of representations } \pi \text { of } \mathcal{M} \text { so that if } \tau \text { is an } \\ \text { irreducible subrepresentation of } \pi, \text { and } \sigma^{\prime} \text { is an irreducible } \mathcal{L}^{\prime}- \\ \text { subrepresentation of the invariants } \tau^{\mathcal{M} \cap \mathcal{U}^{\prime}}, \text { then }\left(\mathcal{L}^{\prime}, \sigma^{\prime}\right) \in \mathscr{L}\end{array}\right.$

In particular, $\mathcal{R}_{\mathcal{G}, \mathscr{L}}=\mathcal{R}_{\mathscr{L}}$. The idempotent $e_{\mathcal{M}, \mathscr{L}}$ is characterized as

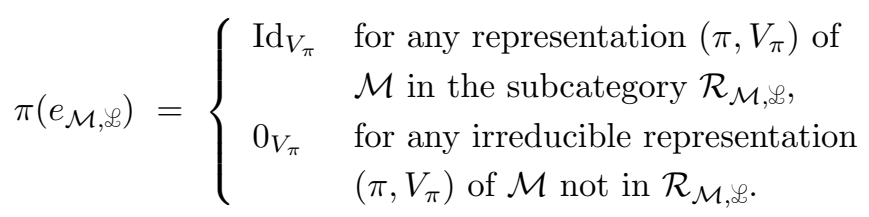

- When the Levi $\mathcal{M}$ does not contain any $\mathcal{G}$-conjugate of $\mathcal{L}$, we set

$$
e_{Q, \mathcal{L}}:=\text { zero function } .
$$

Proposition 3.2.9. Suppose $\mathscr{L}$ is a fixed Harish-Chandra cuspidal class. Let $\mathcal{Q}$ be a parabolic subgroup, and let $\mathcal{Q}=\mathcal{M V}$ be a Levi decomposition. Then

$$
e_{\mathcal{G}, \mathscr{L}} \star e_{\mathcal{V}}=e_{\mathcal{Q}, \mathscr{L}}
$$

Proof. Suppose $\rho$ is any irreducible representation of $\mathcal{G}$. We claim:

$$
\rho\left(e_{\mathcal{G}, \mathscr{L}} \star e_{\mathcal{V}}\right)=\rho\left(e_{\mathcal{Q}, \mathscr{L}}\right) .
$$

Assuming the validity of the claim, we have for all $g \in \mathcal{G}$ that $\rho\left(\delta_{g^{-1}} \star e_{\mathcal{G}, \mathscr{L}} \star e_{\mathcal{V}}\right)=$ $\rho\left(\delta_{g^{-1}} \star e_{\mathcal{Q}, \mathscr{L}}\right)$, so

$$
\operatorname{trace}\left(\rho\left(\delta_{g^{-1}} \star e_{\mathcal{G}, \mathscr{L}} \star e_{\mathcal{V}}\right)\right)=\operatorname{trace}\left(\rho\left(\delta_{g^{-1}} \star e_{\mathcal{Q}, \mathscr{L}}\right)\right) .
$$


Since we can recover any function $f$ on $\mathcal{G}$ as

$$
f(x)=\left(\delta_{x^{-1}} \star f\right)(1)=\frac{1}{\#(\mathcal{G})} \sum_{\rho \in \widehat{\mathcal{G}}} \operatorname{deg}(\rho) \operatorname{trace}\left(\rho\left(\delta_{x^{-1}} \star f\right)\right),
$$

we see (3.2.12), hence (3.2.11) implies the conclusion (3.2.10).

To establish the claim, we note that since $e_{\mathcal{G}, \mathscr{L}}$ is central in the group algebra $\mathbb{C}(\mathcal{G})$, it commutes (under convolution) with $e_{\mathcal{V}}$, and so the operators $\rho\left(e_{\mathcal{G}, \mathscr{L}}\right)$ and $\rho\left(e_{\mathcal{V}}\right)$ commute. Also

$$
\rho\left(e_{\mathcal{G}, \mathscr{L}}\right)= \begin{cases}\operatorname{Id}_{V_{\rho}} & \text { when } \rho \in \mathcal{R}_{\mathscr{L}}, \\ 0_{\mathcal{V}_{\rho}} & \text { otherwise } .\end{cases}
$$

The operator $\rho\left(e_{\mathcal{V}}\right)$ projects to the $\mathcal{V}$-invariants (which we note is a representation of $\mathcal{M})$. Therefore $\rho\left(e_{\mathcal{V}} \star e_{G, \mathscr{L}}\right)$ is the projection to the $\mathcal{V}$-invariants when $\rho \in \mathcal{R}_{\mathscr{L}}$, and is the zero operator otherwise. Since $e_{\mathcal{Q}, \mathscr{L}}=e_{\mathcal{M}, \mathscr{L}} \star e_{\mathcal{V}}$, we see the operator $\rho\left(e_{\mathcal{Q}, \mathscr{L}}\right)$ projects to $\mathcal{R}_{\mathcal{M}, \mathscr{L}}$ when $\rho \in \mathcal{R}_{\mathscr{L}}$ and is zero otherwise. Thus, the claim follows.

We fix a Borel subgroup $\mathcal{B} \subset \mathcal{G}$, and consider the following alternating sum function over $\mathcal{B}$-standard parabolic subgroups :

$$
\sum_{\mathcal{P} \supset \mathcal{B}}(-1)^{\operatorname{rank}(\mathcal{P})} e_{\mathcal{P}, \mathscr{L}}
$$

The next corollary is the finite field result we shall use to show the Bruhat-Tits building version of the above alternating sum belongs to the Bernstein center.

Corollary 3.2.15. Suppose $\mathcal{Q} \supset \mathcal{B}$ is a proper standard parabolic subgroup, i.e, $\mathcal{V}=\operatorname{rad}(\mathcal{Q}) \neq\{1\}$. Then,

$$
\left(\sum_{\mathcal{P} \supset \mathcal{B}}(-1)^{\operatorname{rank}(\mathcal{P})} e_{\mathcal{P}, \mathscr{L}}\right) \star e_{\mathcal{V}}=\text { zero function } .
$$

Proof. For a parabolic $\mathcal{P} \supset \mathcal{B}$, let $\operatorname{rad}(\mathcal{P})$ denote its radical. By the proposition, $e_{\mathcal{P}, \mathscr{L}}=e_{\mathcal{G}, \mathscr{L}} \star e_{\operatorname{rad}(\mathcal{P})}$. Then,

$$
\begin{aligned}
\left(\sum_{\mathcal{P} \supset \mathcal{B}}(-1)^{\operatorname{rank}(\mathcal{P})} e_{\mathcal{P}, \mathscr{L}}\right) & \star e_{\mathcal{V}}=\left(\sum_{\mathcal{P} \supset \mathcal{B}}(-1)^{\operatorname{rank}(\mathcal{P})} e_{\mathcal{G}, \mathscr{L}} \star e_{\operatorname{rad}(\mathcal{P})}\right) \star e_{\mathcal{V}} \\
& =e_{\mathcal{G}, \mathscr{L}} \star\left(\sum_{\mathcal{P} \supset \mathcal{B}}(-1)^{\operatorname{rank}(\mathcal{P})} e_{\operatorname{rad}(\mathcal{P})} \star e_{\mathcal{V}}\right) \\
& =e_{\mathcal{G}, \mathscr{L}} \star 0=0 .
\end{aligned}
$$

More generally, we have the following.

Corollary 3.2.16. Suppose $\mathcal{R} \supsetneq \mathcal{Q}$ are two $\mathcal{B}$-standard parabolic subgroups, and $\mathcal{V}=\operatorname{rad}(\mathcal{Q})$. Then,

$$
\left(\sum_{\mathcal{R} \supset \mathcal{P} \supset \mathcal{B}}(-1)^{\operatorname{rank}(\mathcal{P})} e_{\mathcal{P}, \mathscr{L}}\right) \star e_{\mathcal{V}}=\text { zero function } .
$$

Proof. The proof is similar to the proof of Corollary 3.2.15. We use the property

$$
\left(\sum_{\mathcal{R} \supset \mathcal{P} \supset \mathcal{B}}(-1)^{\operatorname{rank}(\mathcal{P})} e_{\operatorname{rad}(\mathcal{P})}\right) \star e_{\mathcal{V}}=\text { zero function }
$$




\section{The PRojector For the IWAHORI-BERnstein COMPONENT}

We recall our notation: $k$ is a non-archimedean local field, $\mathcal{G}=\mathbf{G}(k)$ is the group of $k$-rational points of a split connected quasisimple group $\mathbf{G}$ defined over $k$. Let $\mathcal{B}=\mathcal{B}(\mathcal{G})$ denote the Bruhat-Tits building of $\mathcal{G}$. We fix a chamber $C_{0} \subset \mathcal{B}$, and let $\mathrm{ht}_{C_{0}}$ be the Bruhat height function on chambers. For $m \in \mathbb{N}$, we define a "ball and shell of radius $m$ " as:

$$
\begin{aligned}
\operatorname{Ball}\left(C_{0}, m\right) & :=\left\{\text { chamber } D \mid \mathrm{ht}_{C_{0}}(D) \leq m\right\}, \\
\operatorname{Shell}\left(C_{0}, m\right) & :=\left\{\text { chambers } D \mid \mathrm{ht}_{C_{0}}(D)=m\right\} .
\end{aligned}
$$

Clearly, $\operatorname{Ball}\left(C_{0}, m\right)$ is a convex simplicial subcomplex of $\mathcal{B}$.

4.1. An equivariant system of idempotents. We define a system of idempotents indexed by the facets of $\mathcal{B}=\mathcal{B}(\mathcal{G})$ as follows:

- For a chamber $F \subset \mathcal{B}$, we take $e_{F}$ to be the idempotent of the trivial representation of the Iwahori subgroup $\mathcal{G}_{F}$.

- For a facet $E \subset \mathcal{B}$, let $F$ be a chamber whose closure contains $E$, so $\mathcal{G}_{E} \supset \mathcal{G}_{F}, \mathcal{G}_{E}^{+} \subset \mathcal{G}_{F}^{+}$, and $\mathcal{B}=\mathcal{G}_{F} / \mathcal{G}_{E}^{+}$is canonically a Borel subgroup of $\mathcal{G}=\mathcal{G}_{E} / \mathcal{G}_{E}^{+}$. The pair $\mathscr{L}=\left(\mathcal{B}, 1_{\mathcal{B}}\right)$ consisting of the trivial representation of the Borel subgroup $\mathcal{B}$ of $\mathcal{G}$ is a Harish-Chandra cuspidal class for $\mathcal{G}$. Let

$$
e_{E}=\text { inflation of the idempotent } e_{\mathcal{B}, \mathscr{L}} \text { of } \mathcal{G} \text { to } \mathcal{G}_{E} \text {. }
$$

The system of idempotents $e_{F}$ for the Iwahori subgroups, i.e., chambers $F$, is clearly $\mathcal{G}$-equivariant, and therefore the collection of canonically attached idempotents $e_{E}$ for arbitrary facets is also $\mathcal{G}$-equivariant.

4.2. Euler-Poincaré presentation of a distribution. The key result we show is the following.

Theorem 4.2.1. For a facet $F$ of $\mathcal{B}(\mathcal{G})$, let $e_{F}$ be the idempotent defined in (4.1.1). The $\mathcal{G}$-invariant distribution defined as the infinite alternating sum

$$
\left(\sum_{E \subset \mathcal{B}(G)}(-1)^{\operatorname{dim}(E)} e_{E}\right)
$$

over the facets is an essentially compact distribution on $\mathcal{G}$, i.e., in the Bernstein center.

To prove Theorem 4.2.1, it is enough to show for any open compact subgroup $J$, and $e_{J}=\frac{1}{\operatorname{meas}(J)} 1_{J}$, the convolution

$$
\sum_{E \subset \mathcal{B}(G)}(-1)^{\operatorname{dim}(E)} e_{E} \star e_{J}
$$

has compact support. Fix a chamber $C_{0} \subset \mathcal{B}$, and let ht $_{C_{0}}$ be the height function, with respect to $C_{0}$, on the chambers of $\mathcal{B}$. When $D$ is a chamber, we use ht $C_{C_{0}}$ to partition the faces of $D$ into the two subsets of parent $p(D)$ faces and child faces $c(D)$ as in (2.3.7). We note $c(D)$ is always non-empty, and when $D \neq C_{0}$, i.e., ht $_{C_{0}}(D)>0$, then $p(D)$ is non-empty too. In this latter situation $\left(\right.$ ht $\left._{C_{0}}(D)>0\right)$, the intersection

$$
D_{+}:=\bigcap_{F \in c(D)} F
$$


is a facet of $D$. Set

$$
\mathcal{F}_{+}(D):=\text { the set of facets } E \text { of } D \text { which contain } D_{+} \text {. }
$$

Theorem 4.2.4. Fix a chamber $C_{0} \subset \mathcal{B}$. Suppose $J$ is an open compact subgroup of $G$. If $D$ is a chamber with $\mathrm{ht}_{C_{0}}(D)$ sufficiently large (depending on $J$ ), then the convolution

$$
\sum_{E \in \mathcal{F}_{+}(D)}(-1)^{\operatorname{dim}(E)} e_{E} \star e_{J}=\text { zero function. }
$$

The proofs are in section 4.4 .

4.3. Some preliminary results. We need several preliminary results to prove Theorem 4.2.4 Suppose $\mathcal{S}$ is a maximal split torus, and $\mathcal{A}=\mathcal{A}(\mathcal{S})$ its associated apartment. Given a choice of positive roots $\Phi^{+} \subset \Phi=\Phi(\mathcal{S})$, we write $\Delta$ for the corresponding set of simple roots, and we write the vectors of the dual basis of $\Delta$ as $\Delta^{\star}=\left\{\lambda_{\alpha} \mid \alpha \in \Delta\right\}$. Fix a chamber $C_{0} \subset \mathcal{A}$. For any choice of a positive root system $\Phi^{+} \subset \Phi$, we defined the $C_{0}$-based sector $S\left(C_{0}, \Phi^{+}\right)$in $\mathcal{A}$ in (2.4.2). The union $\bigcup_{\Phi^{+}} S\left(C_{0}, \Phi^{+}\right)$over all positive sets of roots is the apartment $\mathcal{A}$.

Lemma 4.3.1. Suppose $\Phi^{+} \subset \Phi(\mathcal{S})$ is a set of positive roots and $\Delta \subset \Phi^{+}$are the simple roots. Fix a simple root $\alpha \in \Delta$, and suppose $\gamma \in \Phi^{+}$satisfies $\lambda_{\alpha}(\gamma)>0$, i.e., the expression of $\gamma$ as a sum of simple roots has positive $\alpha$ coefficient. If $D \subset S\left(C_{0}, \Phi^{+}\right)$is a chamber which is separated from $C_{0}$ by $L \geq 2$ distinct affine root hyperplanes $H_{\psi}$ with $\operatorname{grad}(\psi)=\alpha$, then $D$ is separated from $C_{0}$ by at least $L$ distinct affine root hyperplanes $H_{\psi}$ with $\operatorname{grad}(\psi)=\gamma$.

Proof. Let $\gamma=\sum_{\beta \in \Delta} n_{\beta} \beta$ be the linear expansion of $\gamma$ in terms of simple roots. The hypothesis that there are $L$ affine hyperplanes perpendicular to $\alpha$ separating $C_{0}$ and $D$ means $\alpha(y)-\alpha(x)>(L-1)$ for any $x \in \operatorname{recess}\left(C_{0}\right)$, and $y \in \operatorname{recess}(D)$. Suppose $\beta \in \Delta \backslash\{\alpha\}$. If (i) $D$ and $C_{0}$ are separated by an affine hyperplane perpendicular to $\beta$, then $\beta(y)-\beta(x)>0$. Else, (ii) $D$ and $C_{0}$ are not separated by any affine hyperplane perpendicular to $\beta$. Let $Q$ be the set of these simple roots. The set $Q$ is linearly independent (as is any non-empty subset of $\Delta$ ), and therefore we can replace $y$ by some $y^{\prime} \in \operatorname{recess}(D)$, so that $\beta\left(y^{\prime}\right)=\beta(x)$ for all $\beta \in Q$. Therefore, $\gamma\left(y^{\prime}\right)-\gamma(x)=\sum_{\beta \in \Delta} n_{\beta}\left(\beta\left(y^{\prime}\right)-\beta(x)\right)>n_{\alpha}(L-1) \geq(L-1)$. This means $C_{0}$ and $D$ are separated by at least $L$ affine hyperplanes perpendicular to $\gamma$.

Corollary 4.3.2. Suppose $D$ is a chamber in the $C_{0}$-based sector $S\left(C_{0}, \Phi^{+}\right)$, and for all simple roots $\alpha \in \Delta, D$ is separated from $C_{0}$ by $L \geq 2$ affine hyperplanes perpendicular to $\alpha$, then for any $\gamma \in \Phi^{+}, D$ is separated from $C_{0}$ by at least $L$ affine hyperplanes perpendicular to $\gamma$.

Proof. Clear.

\subsection{Proof of Theorems 4.2.4 and 4.2.1, We prove Theorem 4.2.4,}

Proof. We can and do replace the open compact subgroup $J$ by a group $G_{x_{0}, \rho}$, where $x_{0} \in C_{0}$, and $\rho$ is a sufficiently large integer to insure $G_{x_{0}, \rho} \subset J$. The fact that $\rho$ is an integer means $G_{x, \rho}=G_{x_{0}, \rho}$ for any $x \in \operatorname{recess}\left(C_{0}\right)$.

Take $\mathcal{S}$ to be a maximal split torus of $\mathcal{G}$ so that the apartment $\mathcal{A}=\mathcal{A}(\mathcal{S})$ contains $C_{0}$ and $D$. We recall $\mathcal{A}$ is the union of the $C_{0}$-based sectors $S\left(C_{0}, \Phi^{+}\right)$as $\Phi^{+}$runs over the sets of possible positive roots. 
Take $\Phi^{+} \subset \Phi=\Phi(\mathcal{S})$ to be a choice of positive roots so that the chamber based sector $S\left(C_{0}, \Phi^{+}\right)$contains $D$. The choice of the particular set of positive roots may not be unique. For a choice $\Phi_{D}^{+}$, let $\mathbf{B}_{D}^{+}$and $\mathbf{B}_{D}^{-}$be the Borel subgroups of $\mathbf{G}$ associated to the sets of positive roots $\Phi^{+}$and $-\Phi^{+}$. We have a decomposition of the subgroup $J=\mathcal{G}_{x_{0}, \rho}$ into subgroups:

$$
\mathcal{G}_{x_{0}, \rho}=\mathcal{G}_{x_{0}, \rho}^{-} \mathcal{S}_{\rho} \mathcal{G}_{x_{0}, \rho}^{+},
$$

where

$$
\mathcal{G}_{x_{0}, \rho}^{+}:=\mathcal{G}_{x_{0}, \rho} \cap \mathbf{B}_{D}^{+}(k) \text { is a product } \prod_{\substack{\psi, \psi\left(x_{0}\right) \geq \rho \\ \operatorname{grad}(\psi) \in \Phi^{+}}} x_{\psi}
$$

of affine root $\operatorname{groups} \mathcal{X}_{\psi}$, with $\psi\left(x_{0}\right) \geq \rho$, and $\operatorname{grad}(\psi) \in \Phi^{+}$. Similarly,

$$
\mathcal{G}_{x_{0}, \rho}^{-}:=\mathcal{G}_{x_{0}, \rho} \cap \mathbf{B}_{D}^{-}(k)=\prod_{\substack{\psi, \psi\left(x_{0}\right) \geq \rho \\ \operatorname{grad}(\psi) \in-\Phi^{+}}} x_{\psi} .
$$

Suppose $\alpha \in \Phi^{+}$. The intersection $\mathcal{G}_{x_{0}, \rho}^{-} \cap \mathcal{U}_{-\alpha}$ is an affine root group $\chi_{\phi}$ (necessarily $\operatorname{grad}(\phi)=-\alpha$ ), and for all $x \in C_{0}$, we have $\rho+1>\phi\left(x_{0}\right) \geq \rho$. If the chambers $C_{0}$ and $D$ are separated by at least $(\rho+2)$ affine hyperplanes perpendicular to $\alpha$, then $(\rho+1) \leq \phi\left(x_{0}\right)-\phi(y)$, so $\phi(y) \leq-(\rho+1)+\phi\left(x_{0}\right)<0$ for all $y \in \operatorname{recess}(D)$, and thus $\phi(y) \leq 0$ for all $y \in D$. This means the affine root group $X_{\phi}$ contains $\mathcal{G}_{y, 0} \cap \mathcal{U}_{-\alpha}$ for all $y \in D$. We note that since $\phi(x) \geq \rho$ for all $x \in C_{0}$ (so for $x_{0}$ ), it is the case that $X_{\phi}$ is contained in $J=\mathcal{G}_{x_{0}, \rho}$.

To summarize: If $\gamma \in \Phi^{+}$, and $D$ is a chamber of $S\left(C_{0}, \Phi^{+}\right)$separated from $C_{0}$ by sufficiently many (at least $(\rho+2)$ ) affine hyperplanes perpendicular to $\gamma$, then $\mathcal{G}_{x_{0}, \rho}^{-} \cap \mathcal{U}_{-\gamma}$, hence $\mathcal{G}_{x_{0}, \rho}$, contains $\mathcal{G}_{y, 0} \cap \mathcal{U}_{-\gamma}$ for any $y \in D$.

For $k \in \mathbb{N}$, set

$$
R_{k}=\text { the set }\left\{\begin{array}{l}
\text { chambers } D \text { of } S\left(C_{0}, \Phi^{+}\right) \text {satisfying: for each } \alpha \in \Delta, \text { the } \\
\text { chamber } D \text { is separated from } C_{0} \text { by at least } k \text { affine hy- } \\
\text { perplanes } H_{\psi} \text { perpendicular to } \alpha .
\end{array}\right\}
$$

Suppose $D \in \mathcal{R}_{(\rho+2)}$. By Corollary 4.3.2, for any $\gamma \in \Phi^{+}$, the chamber $D$ is separated from $C_{0}$ by at least $(\rho+2)$ affine hyperplanes perpendicular to $\gamma$; hence, $\mathcal{G}_{x_{0}, \rho}$ contains $\mathcal{G}_{y, 0} \cap \mathcal{U}_{-\gamma}$ for all $y \in D$.

When $K$ is a facet in $\mathcal{A}$, define:

$$
\begin{aligned}
\Psi(K) & :=\text { set of affine roots } \psi \text { which vanish on } K, \\
\Psi\left(K, \Phi^{+}\right) & :=\left\{\psi \in \Psi(K) \mid \operatorname{grad}(\psi) \in \Phi^{+}\right\} .
\end{aligned}
$$

Recall $D_{+}$is defined to be the facet of $D$ which is the intersection of all the outward oriented faces of $D$. We pick $y$ to be a point in $\operatorname{recess}\left(D_{+}\right)$. The finite field group $\mathcal{G}_{y, 0} / \mathcal{G}_{y, 0^{+}}$has root system

$$
\Phi\left(\mathcal{G}_{y, 0} / \mathcal{G}_{y, 0^{+}}\right)=\left\{\operatorname{grad}(\psi) \mid \psi \text { affine root so that } \psi_{\left.\right|_{D_{+}}} \equiv 0\right\}=\operatorname{grad}\left(\Psi\left(D_{+}\right)\right) \text {. }
$$

Suppose $\psi \in \Psi\left(D_{+}, \Phi^{+}\right)$. Set $\gamma=\operatorname{grad}(\psi)$. The hypothesis $C_{0}$ and $D$ are separated by $(\rho+2)$ affine hyperplanes perpendicular to $\gamma$ means $\left(\psi(y)-\psi\left(x_{0}\right)\right) \geq(\rho+1)$. Since $\psi(y)=0$, we get $-\psi\left(x_{0}\right) \geq(\rho+1)$. Thus, $\mathcal{G}_{x_{0}, \rho}$ contains $\mathcal{X}_{-\psi}$. This latter 
subgroup is $\mathcal{G}_{y, 0} \cap \mathcal{U}_{-\gamma}$. It follows

$$
V_{y}:=\prod_{\psi \in \Psi\left(D_{+}, \Phi^{+}\right)} x_{-\psi}
$$

is contained in $\mathcal{G}_{x_{0}, \rho}$ and that $V_{y} \mathcal{G}_{D_{+}}^{+}=\mathcal{G}_{D}^{+}$(the subgroup $V_{y}$ adds in the affine root groups $\left.X_{-\psi}\right)$, so $V_{y} \mathcal{G}_{D_{+}}^{+} / \mathcal{G}_{D_{+}}^{+} \subset \mathcal{G}_{D_{+}} / \mathcal{G}_{D_{+}}^{+}$is the unipotent radical of the Borel subgroup $\mathcal{G}_{D} / \mathcal{G}_{D_{+}}^{+}$.

This means, by Corollary 3.2 .15 , the convolution

$$
\begin{aligned}
\sum_{E \in \mathcal{F}_{+}(D)}(-1)^{\operatorname{dim}(E)} e_{E} & \star e_{V_{y}}=\sum_{E \in \mathcal{F}_{+}(D)}(-1)^{\operatorname{dim}(E)}\left(e_{E} \star e_{\mathcal{G}_{y, 0^{+}}}\right) \star e_{V_{y}} \\
& =\sum_{E \in \mathcal{F}_{+}(D)}(-1)^{\operatorname{dim}(E)} e_{E} \star\left(e_{\mathcal{G}_{y, 0^{+}}} \star e_{V_{y}}\right) \\
& =\sum_{E \in \mathcal{F}_{+}(D)}(-1)^{\operatorname{dim}(E)} e_{E} \star\left(e_{\left(\mathcal{G}_{y, 0}+V_{y}\right)}\right) \\
& =\text { zero function. }
\end{aligned}
$$

So, under the assumption $D \subset R_{(\rho+2)}$, we see

$$
\begin{aligned}
\sum_{E \in \mathcal{F}_{+}(D)}(-1)^{\operatorname{dim}(E)} e_{E} & \star e_{\mathcal{G}_{x_{0}, \rho}}=\sum_{E \in \mathcal{F}_{+}(D)}(-1)^{\operatorname{dim}(E)} e_{E} \star\left(e_{V_{y}} \star e_{\mathcal{G}_{x_{0}, \rho}}\right) \\
& =\left(\sum_{E \in \mathcal{F}_{+}(D)}(-1)^{\operatorname{dim}(E)} e_{E} \star e_{V_{y}}\right) \star e_{\mathcal{G}_{x_{0}, \rho}} \\
& =0 \star e_{\mathcal{G}_{x_{0}, \rho}}=\text { zero function. }
\end{aligned}
$$

We turn to the situation when the chamber $D$ is in $S\left(C_{0}, \Phi^{+}\right) \backslash R_{(\rho+2)}$.

For a subset $I \subset \Delta$, and an integer $k$ set

$$
\begin{aligned}
\mathcal{R}_{\{I, k\}}:=\left\{D \subset S\left(C_{0}, \Phi^{+}\right) \mid \mathrm{ht}_{C_{0}}^{ \pm \alpha}(D)\right. & \geq k \forall \alpha \in I, \text { and } \\
\operatorname{ht}_{C_{0}}^{ \pm \alpha}(D) & <k \forall \alpha \in(\Delta \backslash I)\} .
\end{aligned}
$$

We note

(i) $R_{\{\Delta, k\}}$ is the set $R_{k}$ in (4.4.1).

(ii) The set $R_{\{I, k\}}$ is finite precisely when $I=\emptyset$.

(iii) For a fixed $k$, the sets $R_{\{I, k\}}$ partition $S\left(C_{0}, \Phi^{+}\right)$.

To complete the proof of Theorem 4.2.4, we need to show, when $I$ is non-empty, the convolution (4.2.5) vanishes for all $D \in \mathcal{R}_{\{I,(\rho+2)\}}$ provided $h_{C_{0}}(D)$ is sufficiently large. The case $I=\Delta$ has already been treated above. Set

$\Phi^{+}\left(D_{+}, I\right):=\left\{\gamma \in \operatorname{grad}\left(\Psi\left(D_{+}, \Phi^{+}\right)\right) \mid\right.$there exists $\alpha \in I$, such that $\left.\lambda_{\alpha}(\gamma)>0\right\}$. We recall the $\mathcal{S}_{c}$-roots $\Phi\left(\mathcal{G}_{D_{+}} / \mathcal{G}_{D_{+}}^{+}, \mathcal{S}_{c}\right)$ of the finite field group $\mathcal{G}_{D_{+}} / \mathcal{G}_{D_{+}}^{+}$are the gradients of the affine roots in the set $\Psi\left(D_{+}\right)$(see (4.4.2)). Suppose $\psi \in \Psi\left(D_{+}\right)$ is such an affine root, and $\gamma:=\operatorname{grad}(\psi) \in \Phi^{+}$. If there is an $\alpha \in I$ such that $\lambda_{\alpha}(\gamma)>0$, i.e., $\operatorname{grad}(\psi) \in \Phi^{+}\left(D_{+}, I\right)$, then, by Lemma 4.3.1 necessarily $C_{0}$ and $D$ are separated by at least $\rho+1$ affine hyperplanes perpendicular to $\gamma$. Take $y \in \operatorname{recess}\left(D_{+}\right)$. Since $\psi(y)=0$, we get $-\psi\left(x_{0}\right)=\left(\psi(y)-\psi\left(x_{0}\right)\right)>\rho$. This 
means both $x_{-\psi} \subset \mathcal{G}_{x_{0}, \rho}$, as well as the image of $x_{-\psi}$ in the finite field group $\mathcal{G}_{y, 0} / \mathcal{G}_{y, 0}^{+}$(equal to $\mathcal{G}_{D_{+}} / \mathcal{G}_{D_{+}}^{+}$) is the (non-trivial) root group attached to $-\gamma$. Thus,

$$
\mathcal{G}_{x_{0}, \rho} \supset V_{y}:=\prod_{\substack{\psi \in \Psi\left(D_{+}\right) \\ \operatorname{grad}(\psi) \in \Phi^{+}\left(D_{+}, I\right)}}\left(\mathcal{G}_{y, 0} \cap \mathcal{U}_{-\gamma}\right) .
$$

Recall, the set $\left\{\lambda_{\alpha} \mid \alpha \in \Delta\right\}$ is the set of fundamental weights. We set

$$
\lambda_{I}:=\sum_{\alpha \in I} \lambda_{\alpha}
$$

Let $F$ be the facet of $D$ so that the $\mathcal{G}_{F} / \mathcal{G}_{D_{+}}^{+}$is the standard parabolic whose standard Levi is generated (modulo $\mathcal{G}_{D_{+}}^{+}$) by the affine root groups $X_{\psi}$ with $\psi \in$ $\Psi\left(D_{+}\right)$satisfying $\lambda_{I}(\operatorname{grad}(\psi))=0$, and whose unipotent radical is the product (modulo $\mathcal{G}_{D_{+}}^{+}$) of the root groups $X_{-\psi}$ with $\psi \in \Psi\left(D_{+}\right)$and $\lambda_{I}(\operatorname{grad}(\psi))>0$, i.e., $V_{y} \mathcal{G}_{D_{+}}^{+} / \mathcal{G}_{D_{+}}^{+}$. Provided $V_{y} \mathcal{G}_{D_{+}}^{+} / \mathcal{G}_{D_{+}}^{+}$is not the trivial unipotent subgroup, the ending argument for the case $\mathcal{R}_{(\rho+2)}$ can be applied to deduce the convolution (4.2.5) vanishes. By what we have argued above, this happens if there exists $\psi \in$ $\Psi\left(D_{+}, \Phi^{+}\right)$and an $\alpha \in I$ with $\lambda_{\alpha}(\operatorname{grad}(\psi)) \neq 0$. For these chambers the convolution (4.2.5) vanishes.

Given a subset $K \subset \Delta$, set

(4.4.8) $\Phi(K):=\{\alpha \in \Phi \mid \alpha$ is a linear combination of (simple) roots in $K\}$.

We are reduced to investigating $D \in \mathcal{R}_{\{I,(\rho+2)\}}$ so that every $\psi \in \Psi\left(D_{+}, \Phi^{+}\right)$ satisfies $\operatorname{grad}(\psi) \in \Phi(\Delta \backslash I)$. Denote this set by $\mathcal{R}_{I,(\rho+2)}^{\text {last }}$; so,

$\mathcal{R}_{\{I,(\rho+2)\}}^{\text {last }}:=\left\{D \in \mathcal{R}_{\{I,(\rho+2)\}} \mid \psi \in \Psi\left(D_{+}, \Phi^{+}\right)\right.$satisfies $\left.\operatorname{grad}(\psi) \in \Phi(\Delta \backslash I)\right\}$.

The set of outward oriented faces of a chamber $D \in \mathcal{R}_{\{I,(\rho+2)\}}^{\text {last }}$ must have gradients in $\Phi(\Delta \backslash I)$, but in principle, it could be a proper subset. We partition $\mathcal{R}_{\{I,(\rho+2)\}}^{\text {last }}$ as follows: To a (non-empty) subset $K$ of $\Delta \backslash I$, we set

$\mathcal{R}_{\{I,(\rho+2)\}}^{\mathrm{K}}:=\left\{D \in \mathcal{R}_{\{I,(\rho+2)\}} \mid \psi \in \Psi\left(D_{+}, \Phi^{+}\right)\right.$satisfies

(i) $\operatorname{grad}(\psi) \in \Phi(\Delta \backslash I)$

(ii) each simple root $\beta \in K$ occurs as a $\operatorname{grad}(\psi)\}$.

The sets $\mathcal{R}_{\{I,(\rho+2)\}}^{\mathrm{K}}$ are a partitioning of $\mathcal{R}_{\{I,(\rho+2)\}}^{\text {last }}$ into $2^{\#(\Delta \backslash I)}-1$ subsets. Furthermore, a chamber in $\mathcal{R}_{\{I,(\rho+2)\}}^{\mathrm{K}}$ is incident with the permissible set $\tilde{K}:=\{\psi \in$ $\left.\Psi\left(D_{+}, \Phi^{+}\right) \mid \operatorname{grad}(\psi) \in K\right\}$ (see (2.5.3)$)$. We apply Proposition 2.5.5 to say the number of chambers in $\mathcal{R}_{\{I,(\rho+2)\}}^{\mathrm{K}}$ is finite. So, $\mathcal{R}_{\{I,(\rho+2)\}}^{\text {last }}$ is finite, and we deduce the Theorem 4.2.4 when $D$ is a chamber in $\mathcal{A}$ with $\mathrm{ht}_{C_{0}}(D)$ sufficiently large.

The (compact) Iwahori subgroup $\mathcal{G}_{C_{0}}$ acts transitively on the set $\mathcal{A}\left(C_{0}\right)$ of apartments containing $C_{0}$. Fix an apartment $\mathcal{A}^{\prime}$ containing $C_{0}$. Consider an apartment $g \mathcal{A}^{\prime}\left(g \in \mathcal{G}_{C_{0}}\right)$. The above argument applied to the apartment $g \mathcal{A}^{\prime}$ shows there is a compact open subgroup $K_{g}$ and an integer $M_{g}>0$ so that the convolution (4.2.5) vanishes for all $D \in h g \mathcal{A}^{\prime}\left(h \in K_{g}\right)$ provided $h_{C_{0}}(D) \geq M_{g}$. The collection 
of sets $\left\{K_{g} g \mid g \in \mathcal{G}_{C_{0}}\right\}$ is an open cover of $\mathcal{G}_{C_{0}}$, and so has a finite subcover $\left\{g_{i} K_{g_{i}} \mid i=1, \ldots, n\right\}$. Take $M=\max \left(M_{g_{1}}, \ldots, M_{g_{n}}\right)$. The convolution (4.2.5) vanishes for any chamber $D$ of $\mathcal{B}$ satisfying ht $C_{0}(D) \geq M$.

We turn to the proof of Theorem 4.2.1.

Proof. We recall that we can replace the open compact subgroup by a subgroup $\mathcal{G}_{x_{0}, \rho} \subset J$ with $x_{0} \in C_{0}$ and $\rho$ integral. We fix a chamber $C_{0}$ and for a positive integer $m$, consider the ball Ball $\left(C_{0}, m\right)$ of (4.0.1). We consider the convolutions

$$
\sum_{E \subset \operatorname{Ball}\left(C_{0}, m\right)}(-1)^{\operatorname{dim}(E)} e_{E} \star e_{\mathcal{G}_{x_{0}, \rho}} .
$$

The sum is over the facets in $\operatorname{Ball}\left(C_{0}, m\right)$. It is clear that the convolution over $\operatorname{Ball}\left(C_{0},(m+1)\right)$ is obtained from the convolution over $\operatorname{Ball}\left(C_{0}, m\right)$ by adding convolution terms of the form

$$
\sum_{E \in \mathcal{F}_{+}(D)}(-1)^{\operatorname{dim}(E)} e_{E} \star e_{\mathcal{G}_{x_{0}, \rho}},
$$

where $D$ runs over the chambers satisfying ht $_{C_{0}}(D)=(m+1)$, i.e., in $\operatorname{Shell}\left(C_{0},(m+\right.$ 1)). By Theorem 4.2.4, these convolution terms vanish provided $m$ is sufficiently large. Therefore, the convolution over $\operatorname{Ball}\left(C_{0}, m\right)$ and $\operatorname{Ball}\left(C_{0},(m+1)\right)$ are the same when $m$ is sufficiently large. This establishes Theorem 4.2.1.

4.5. The Iwahori-Bernstein component. The next proposition and corollary show the essentially compact distribution of Theorem 4.2.1 is the projector to the Bernstein component of representations with a non-zero Iwahori fixed vector.

Proposition 4.5.1. For any facet $E \subset \mathcal{B}=\mathcal{B}(G)$, define the idempotent $e_{E}$ as in (4.1.1). Fix a chamber $C_{0}$ in $\mathcal{B}=\mathcal{B}(G)$. Then:

(i)

$$
\sum_{E \subset C_{0}}(-1)^{\operatorname{dim}(E)} e_{E} \star e_{C_{0}}=e_{C_{0}}
$$

(ii) For any chamber $D \neq C_{0}$ :

$$
\sum_{E \in \mathcal{F}_{+}(D)}(-1)^{\operatorname{dim}(E)} e_{E} \star e_{C_{0}}=\text { zero function } .
$$

(iii)

$$
\left(\sum_{E \subset \mathcal{B}(G)}(-1)^{\operatorname{dim}(E)} e_{E}\right) \star e_{C_{0}}=e_{C_{0}}
$$

Proof. Statement (i) follows from the fact that $e_{E} \star e_{C_{0}}=e_{C_{0}}$ for any facet $E \subset C_{0}$.

Statement (ii) is seen by modifying the proof of Theorem 4.2.4. Let $\Phi^{+} \subset \Phi$ be a positive root system so that $S\left(C_{0}, \Phi^{+}\right)$contains $D$. The difference between the two Iwahori subgroups $\mathcal{G}_{D}$ and $\mathcal{G}_{C_{0}}$ is the following: for $\alpha \in \Phi^{+}$it is the 
case $\left(\mathcal{G}_{C_{0}} \cap \mathcal{U}_{\alpha}\right) \subset\left(\mathcal{G}_{D} \cap \mathcal{U}_{\alpha}\right)$, while $\left(\mathcal{G}_{C_{0}} \cap \mathcal{U}_{-\alpha}\right) \supset\left(\mathcal{G}_{D} \cap \mathcal{U}_{-\alpha}\right)$. Thus, $\left(\mathcal{G}_{C_{0}, 0^{+}} \cap\right.$ $\left.\mathcal{G}_{D_{+}, 0}\right) \mathcal{G}_{D_{+}, 0^{+}}=\mathcal{G}_{D}$. So

$$
\begin{aligned}
& \sum_{E \in \mathcal{F}_{+}(D)}(-1)^{\operatorname{dim}(E)} e_{E} \star e_{C_{0}} \\
& =\left(\sum_{E \in \mathcal{F}_{+}(D)}(-1)^{\operatorname{dim}(E)} e_{E} \star e_{\mathcal{G}_{D_{+}, 0^{+}}}\right) \star\left(e_{\left(\mathcal{G}_{C_{0}, 0} \cap \cap \mathcal{G}_{D_{+}, 0}\right)} \star e_{C_{0}}\right) \\
& =\left(\sum_{E \in \mathcal{F}_{+}(D)}(-1)^{\operatorname{dim}(E)} e_{E} \star\left(e_{\mathcal{G}_{D_{+}, 0^{+}}} \star e_{\left(\mathcal{G}_{C_{0}, 0} \cap \cap \mathcal{G}_{D_{+}, 0}\right)}\right)\right) \star e_{C_{0}} \\
& =\left(\sum_{E \in \mathcal{F}_{+}(D)}(-1)^{\operatorname{dim}(E)} e_{E} \star e_{\mathcal{G}_{D}}\right) \star e_{C_{0}} \\
& =\text { zero function. }
\end{aligned}
$$

Statement (iii) is an obvious consequence of statements (i) and (ii).

Corollary 4.5.2. The distribution $P:=\left(\sum_{E \subset \mathcal{B}(G)}(-1)^{\operatorname{dim}(E)} e_{E}\right)$ is the projector to the Bernstein component with non-zero Iwahori fixed vectors.

Proof. Suppose $\left(\pi, V_{\pi}\right)$ is an irreducible smooth representation of $G$. The operator $\pi(P)$ is a scalar operator.

For any facet $E \subset \mathcal{B}$, the operator $\pi\left(e_{E}\right)$ projects to the subspace $V_{\pi}^{\mathcal{G}_{E, 0^{+}}}$. Furthermore, from the definition of $e_{E}$, any non-zero irreducible representation of $\mathcal{G}_{E, 0} / \mathcal{G}_{E, 0^{+}}$in $V_{\pi}^{\mathcal{G}_{E, 0^{+}}}$must have a non-zero Iwahori fixed vector for any Iwahori subgroup $\mathcal{G}_{D}$ contained in $\mathcal{G}_{E}$. It follows that if $V_{\pi}^{\mathcal{G}_{D}}=\{0\}$ for any chamber $D$, then the scalar $\pi(P)$ is zero, i.e., a necessary condition for $\pi(P)$ to be non-zero is that $\pi$ has a non-zero Iwahori fixed vector.

On the other hand, if there is a chamber $D$, so that $V_{\pi}^{\mathcal{G}_{D}} \neq\{0\}$, then $V_{\pi}^{\mathcal{G}_{0}} \neq\{0\}$ too. Since $P \star e_{C_{0}}=e_{C_{0}}$, we conclude $\pi(P)=\operatorname{Id}_{V_{\pi}}$, and thus $P$ is the Bernstein projector for the component with non-zero Iwahori fixed vectors.

\section{General Depth Zero}

5.1. Preliminaries. Suppose $F$ is a facet of $\mathcal{B}$. Let $\mathcal{G}_{F}$ be the parahoric subgroup attached to $F\left[\mathrm{BTb}\right.$, i.e., the $\mathfrak{O}_{k}$-rational points of the identity component of the $\mathfrak{O}_{k}$-group scheme of the fixer group of $F$ (which fixes each point $x \in F$ ). The quotient $\mathcal{G}_{F} / \mathcal{G}_{F}^{+}$is the group of $\mathbb{F}_{q}$-rational points of a reductive linear connected group. We will take a cuspidal representation $\sigma$ of $\mathcal{G}_{F} / \mathcal{G}_{F}^{+}$, and use the corresponding idempotent $e_{\sigma}$ of $\mathcal{G}_{F}$ to define a natural sum of Bernstein projectors of $\mathcal{G}$. In order to do this we recall some preliminaries.

To a parahoric subgroup $\mathcal{G}_{F}$, we can attach a Levi subgroup $\mathcal{M} \subset \mathcal{G}$. We recall some results from section 6.2 in $[\mathrm{MPb}$. We take a maximal split $k$-torus $\mathcal{S}$ so that the apartment $\mathcal{A}(\mathcal{S})$ contains the facet $F$. Then $\mathcal{S}$ gives rise to a maximal split $\mathbb{F}_{q}$-torus in $\mathcal{G}_{F} / \mathcal{G}_{F}^{+}$. We take the unique $k$-subtorus $\mathcal{C}$ of $\mathcal{S}$ so that $\left(\mathcal{C} \cap \mathcal{G}_{F}\right) /\left(\mathcal{C} \cap \mathcal{G}_{F}^{+}\right)$ is the center of $\mathcal{G}_{F} / \mathcal{G}_{F}^{+}$. The centralizer

$$
\mathcal{M}=Z_{\mathcal{G}}(\mathcal{C})
$$

is a Levi subgroup with center $\mathcal{C}$, and $\mathcal{M}_{F}=\mathcal{M} \cap \mathcal{G}_{F}$ is a maximal parahoric of $\mathcal{M}$, and $\mathcal{G}_{F} / \mathcal{G}_{F}^{+}=\mathcal{M}_{F} / \mathcal{M}_{F}^{+}$. The torus $\mathcal{S}$, hence $\mathcal{M}$ is defined up to a conjugation 
by $\mathcal{G}_{F}$. Let $\mathcal{F}_{F}=N_{\mathcal{M}}\left(\mathcal{M}_{F}\right)(\supset \mathcal{C})$ denote the normalizer of $\mathcal{M}_{F}$ in $\mathcal{M}$. The quotient group $\mathcal{F}_{F} / \mathcal{C}$ is compact.

We view an irreducible cuspidal representation $\sigma$ of the finite field group $\mathcal{G}_{F} / \mathcal{G}_{F}^{+}$ as also one of $\mathcal{M}_{F} / \mathcal{M}_{F}^{+}$, and we continue to write $\sigma$ for its inflation to an irreducible representation of $\mathcal{M}_{F}$. Let

$$
\Sigma(F, \sigma):=\left\{\begin{array}{l}
\text { the collection of those irreducible representations of } \mathcal{F}_{F}, \\
\text { up to equivalence, whose restrictions to } \mathcal{M}_{F} \text { contains } \sigma .
\end{array}\right.
$$

Define $\tau_{1}, \tau_{2} \in \Sigma(F, \sigma)$ to be equivalent if there is an unramified character $\chi$ of $\mathcal{M}$ so that $\tau_{2}=\tau_{1} \otimes \chi_{\left.\right|_{\mathcal{F}_{F}}}$. This equivalence relation partitions the collection $\Sigma(F, \sigma)$ into finitely many equivalence classes. We recall the following from [MPb].

Proposition 5.1.3 (Proposition 6.6 in $[\mathrm{MPb}]$ ). $\quad$ Suppose $F \subset \mathcal{B}(\mathcal{G})$ is a facet and $\mathcal{M}$ is a Levi subgroup attached to $F$ as in (5.1.1), so $\mathcal{G}_{F} / \mathcal{G}_{F}^{+}=\mathcal{M}_{F} / \mathcal{M}_{F}^{+}$, and suppose $\sigma$ is the inflation to $\mathcal{M}_{F}$ of an irreducible cuspidal representation of $\mathcal{M}_{F} / \mathcal{M}_{F}^{+}$.

- Given $\tau \in \Sigma(F, \sigma)$, the representation $\mathrm{c}-\operatorname{Ind}_{\mathcal{F}_{F}}^{\mathcal{M}}(\tau)$ is a cuspidal representation of $\mathcal{M}$.

- Suppose $\left(\pi, V_{\pi}\right)$ is an irreducible smooth representation of $\mathcal{M}$ which contains $\sigma$ upon restriction to $\mathcal{M}_{F}$. Then $\pi$ is equivalent to $\mathrm{c}-\operatorname{Ind}_{\mathcal{F}_{F}}^{\mathcal{M}}(\tau)$ for some $\tau \in \Sigma(F, \sigma)$.

Proposition 5.1.4 (Proposition 5.3 in [MPb]). Suppose:

- $F$ and $E$ are two facets of $\mathcal{B}(\mathcal{G})$, and $\tau$ and $\kappa$ are irreducible representations of $\mathcal{G}_{F}$ and $\mathcal{G}_{E}$ inflated from cuspidal representations of $\mathcal{G}_{F} / \mathcal{G}_{F}^{+}$and $\mathcal{G}_{E} / \mathcal{G}_{E}^{+}$, respectively.

- $\left(\pi, V_{\pi}\right)$ is a smooth irreducible representation of $\mathcal{G}$ so that $\tau$ and $\kappa$ appear in the restriction of $\pi$ to $\mathcal{G}_{F}$ and $\mathcal{G}_{E}$, respectively.

Then, there exists $g \in G$ so that $\mathcal{G}_{F} \cap \mathcal{G}_{g E}$ surjects onto both $\mathcal{G}_{F} / \mathcal{G}_{F}^{+}$and $\mathcal{G}_{g E} / \mathcal{G}_{g E}^{+}$, and $\kappa=\tau \circ \operatorname{Ad}(g)$.

We note the fact that $\mathcal{G}_{F} \cap \mathcal{G}_{g E}$ surjects to $\mathcal{G}_{F} / \mathcal{G}_{F}^{+}$and $\mathcal{G}_{g E} / \mathcal{G}_{g E}^{+}$means there is a canonical identification of these latter two groups, and hence a canonical way to any representation of one as also a representation of the other.

Proposition 5.1.5 (Proposition 6.2 in [MPb]). Suppose $F, E \subset \mathcal{B}(\mathcal{G})$ are facets so that $\mathcal{G}_{F} \cap \mathcal{G}_{E}$ surjects onto $\mathcal{G}_{F} / \mathcal{G}_{F}^{+}$and $\mathcal{G}_{E} / \mathcal{G}_{E}^{+}$, respectively, and $\sigma$ is an irreducible cuspidal representation of $\mathcal{G}_{F} / \mathcal{G}_{F}^{+}=\mathcal{G}_{E} / \mathcal{G}_{E}^{+}$. Let $\sigma_{F}$ and $\sigma_{E}$ denote, respectively, the inflation of $\sigma$ to $\mathcal{G}_{F}$ and $\mathcal{G}_{E}$. If $\left(\pi, V_{\pi}\right)$ is an irreducible smooth representation of $\mathcal{G}$ which contains $\sigma_{F}$ upon restriction to $\mathcal{G}_{F}$, then $\pi$ also contains $\sigma_{E}$ upon restriction to $\mathcal{G}_{E}$.

Recall from section 3.4 in [MPb], two parahoric subgroups $\mathcal{G}_{F}$ and $\mathcal{G}_{E}$ are associate if there exists $g \in \mathcal{G}$ so that $\left(\mathcal{G}_{F} \cap \mathcal{G}_{g E}\right)$ surjects onto both $\mathcal{G}_{F} / \mathcal{G}_{F}^{+}$and $\mathcal{G}_{g E} / \mathcal{G}_{g E}^{+}$. In this situation, we have a canonical identification $\mathcal{G}_{F} / \mathcal{G}_{F}^{+}=\mathcal{G}_{g E} / \mathcal{G}_{g E}^{+}$. We define a cuspidal pair $(E, \sigma)$ to be a facet $E$, and a cuspidal representation of $\mathcal{G}_{E} / \mathcal{G}_{E}^{+}$(inflated to $\mathcal{G}_{E}$ ).

If $E$ is a facet in an apartment $\mathcal{A}$, let $\mathcal{A S}(\mathcal{A}, E)$ denote the minimal affine subspace of $\mathcal{A}$ which contains $E$. It is equal to the intersection of all the affine hyperplanes 
$H_{ \pm \psi}\left(\right.$ of $\mathcal{A}$ ) which contain $E$. We observe that if $E$ and $F$ belong to $\mathcal{A}$, then $\mathcal{G}_{F} \cap \mathcal{G}_{E}$ surjects onto both $\mathcal{G}_{F} / \mathcal{G}_{F}^{+}$and $\mathcal{G}_{E} / \mathcal{G}_{E}^{+}$precisely when $\mathcal{A S}(\mathcal{A}, E)=\mathcal{A S}(\mathcal{A}, F)$.

\section{Lemma 5.1.6.}

- Associativity of parahoric subgroups in $\mathcal{G}$ is an equivalence relation.

- If $F, E \subset \mathcal{B}(\mathcal{G})$ are associate facets, then the Levi subgroups attached to them by the above procedure are conjugate in $\mathcal{G}$.

Proof. To prove the first statement, it suffices to prove transitivity. Suppose facets $F_{a}$, and $F_{b}$ are associate. Take $g \in \mathcal{G}$ so that $\left(\mathcal{G}_{F_{a}} \cap \mathcal{G}_{g F_{b}}\right)$ surjects onto $\mathcal{G}_{F_{a}} / \mathcal{G}_{F_{a}}^{+}$and $\mathcal{G}_{g F_{b}} / \mathcal{G}_{g F_{b}}^{+}$. This is equivalent to the equality of $\mathcal{A S}\left(\mathcal{A}, F_{a}\right)$ and $\mathcal{A S}\left(\mathcal{A}, F_{g b}\right)$ for any apartment $\mathcal{A}$ containing both facets. Similarly, suppose $F_{b}$ and $F_{c}$ are associate. This means there is an $h \in \mathcal{G}$ so that $\left(\mathcal{G}_{F_{b}} \cap \mathcal{G}_{h F_{c}}\right)$ surjects onto $\mathcal{G}_{F_{b}} / \mathcal{G}_{F_{b}}^{+}$and $\mathcal{G}_{h F_{c}} / \mathcal{G}_{h F_{c}}^{+}$. Hence $\left(\mathcal{G}_{g F_{b}} \cap \mathcal{G}_{g h F_{c}}\right)$ surjects onto $\mathcal{G}_{g F_{b}} / \mathcal{G}_{g F_{b}}^{+}$and $\mathcal{G}_{g h F_{c}} / \mathcal{G}_{g h F_{c}}^{+}$. Choose a chamber $C$ in $\mathcal{A}$ which contains the facet $g F_{b}$. The Iwahori subgroup $\mathcal{G}_{C}$ acts transitively on the apartments containing $C$, so there is a $k \in \mathcal{G}_{C}$ satisfying $k\left(g h F_{c}\right) \subset \mathcal{A}$. In $\mathcal{A}$, we have,

$$
\mathcal{A S}\left(\mathcal{A}, F_{a}\right)=\mathcal{A S}\left(\mathcal{A},\left(g F_{b}\right)\right)=\mathcal{A S}\left(\mathcal{A},\left(k g F_{b}\right)\right)=\mathcal{A S}\left(\mathcal{A},\left(k g h F_{c}\right)\right),
$$

which means $F_{a}$ and $F_{c}$ are associate.

The second assertion follows from the first. This is because the Levi subgroup attached to $F$ is the centralizer $C_{\mathcal{G}}(\mathcal{Z})$ of a lift $\mathcal{Z}(\subset \mathcal{S})$ of the central torus $\mathcal{Z}$ of the finite field group $\mathcal{G}_{F} / \mathcal{G}_{F}^{+}$. If $E$ and $F$ are associate, we can assume $\left(\mathcal{G}_{F} \cap \mathcal{G}_{E}\right)$ surjects onto $\mathcal{G}_{F} / \mathcal{G}_{F}^{+}$and $\mathcal{G}_{E} / \mathcal{G}_{E}^{+}$, and thereby canonically identify the two, and therefore their central torus, and hence lift. The assertion follows.

We recall the equivalence relation in the data used to define a Bernstein component: Suppose $\mathcal{M}$ is a parabolic $k$-subgroup of $\mathcal{G}$, and $\pi_{a}$ and $\pi_{b}$ are two irreducible cuspidal representations of $\mathcal{M}$. Define

$$
\pi_{a} \sim \pi_{b} \quad\left\{\begin{array}{l}
\text { when there is a } g \in N_{\mathcal{G}}(\mathcal{M}) \text { so that the representation } \\
\pi_{a}^{g}:=\pi_{a} \circ \operatorname{Ad}(g) \text { is isomorphic to the representation } \pi_{b} .
\end{array}\right.
$$

Lemma 5.1.8. $\quad$ Suppose $\mathcal{M}$ is a parabolic subgroup of $\mathcal{G}$, and $F, E \subset \mathcal{B}(\mathcal{G})$ are facets contained in $\mathcal{B}(\mathcal{N})$ so that $\mathcal{M} \cap \mathcal{G}_{F}$ and $\mathcal{M} \cap \mathcal{G}_{E}$ are maximal parahoric subgroups of $\mathcal{M}$ and

$$
\pi_{a}=\mathrm{c}-\operatorname{Ind}_{\mathcal{F}_{F}}^{\mathcal{M}}(\tau), \quad \pi_{b}=\mathrm{c}-\operatorname{Ind}_{\mathcal{F}_{E}}^{\mathcal{\mathcal { N }}}(\kappa),
$$

are equivalent irreducible cuspidal representations $\left(\pi_{a} \sim \pi_{b}\right)$. Then, the facets $F, E$ are associate.

Proof. We note that $\pi_{a} \circ \operatorname{Ad}(g)=\mathrm{c}-\operatorname{Ind}_{g^{-1} \mathcal{F}_{F} g}^{\mathcal{M}}(\tau \circ \operatorname{Ad}(g))=\mathrm{c}-\operatorname{Ind}_{\mathcal{F}_{g^{-1}} F}^{\mathcal{M}}(\tau \circ \operatorname{Ad}(g))$. Thus, the hypothesis that $\pi_{a}^{g}$ and $\pi_{b}$ are isomorphic representations of $\mathcal{M}$ means there is $h \in \mathcal{M}$ so that $\mathcal{F}_{g^{-1} F}=h \mathcal{F}_{E} h^{-1}$ and $\kappa=\tau \circ \operatorname{Ad}(g) \circ \operatorname{Ad}(h)$. In particular, this means the two facets $F, E \subset \mathcal{B}(\mathcal{G})$ are associate.

5.2. Bernstein components. Suppose $F$ is a facet in $\mathcal{B}(\mathcal{G})$, and $\sigma$ is the inflation to $\mathcal{G}_{F}$ of an irreducible cuspidal representation of $\mathcal{G}_{F} / \mathcal{G}_{F}^{+}$, i.e., in the terminology of the introduction, $(F, \sigma)$ is a cuspidal pair. Let $\mathcal{M}$ be a Levi subgroup as in (5.1.1). As mentioned there, the group $\mathcal{M}_{F}=\left(\mathcal{M} \cap \mathcal{G}_{F}\right)$ is a maximal parahoric subgroup of $\mathcal{M}$. The unramified characters of $\mathcal{M}$ act by twists on $\Sigma(F, \sigma)$. There 
are finitely many orbits. We take $\tau \in \Sigma(F, \sigma)$, and consider the irreducible cuspidal representation $\pi(\tau)=\mathrm{c}-\operatorname{Ind}_{\mathcal{F}_{F}}^{\mathcal{M}}(\tau)$ (of the Levi $\mathcal{M}$ ), and the Bernstein component $\Omega([\mathcal{M}, \pi(\tau)])$. Denote by

$$
P(F, \sigma)
$$

the sum of the Bernstein projectors of the finitely many Bernstein components obtained from $\tau \in \Sigma(F, \sigma)$.

Lemma 5.2.2. Suppose $(F, \sigma)$ is a cuspidal pair and $E$ is a facet which is contained in facets $F_{1}$ and $F_{2}$ which are associates to $F$ (say $g_{1}, g_{2} \in \mathcal{G}$ so that $\mathcal{G}_{F_{1}} \cap \mathcal{G}_{g_{1} F}$ surjects to $\mathcal{G}_{F_{1}} / \mathcal{G}_{F_{1}}^{+}$and $\mathcal{G}_{g_{1} F} / \mathcal{G}_{g_{1} F}^{+}$, and $\mathcal{G}_{F_{2}} \cap \mathcal{G}_{g_{2} F}$ surjects to $\mathcal{G}_{F_{2}} / \mathcal{G}_{F_{2}}^{+}$ and $\left.\mathcal{G}_{g_{2} F} / \mathcal{G}_{g_{2} F}^{+}\right)$. Then, $\rho \in \widehat{\mathcal{G}_{E} / \mathcal{G}_{E}^{+}}$contains $\operatorname{Ad}\left(g_{1}\right) \sigma$ if and only if it contains $\operatorname{Ad}\left(g_{2}\right) \sigma$.

We recall the fact that $\mathcal{G}_{F_{i}} \cap \mathcal{G}_{g_{i} F}$ surjects to $\mathcal{G}_{F_{i}} / \mathcal{G}_{F_{i}}^{+}$and $\mathcal{G}_{g_{i} F} / \mathcal{G}_{g_{i} F}^{+}$means there is a canonical identification of these latter two groups, and hence a canonical way to view $\operatorname{Ad}\left(g_{i}\right) \sigma$ as a representation of $\mathcal{G}_{F_{i}}$.

Proof. We easily reduce to the case that $F_{1}=F$; so, suppose $\rho \in \widehat{\mathcal{G}_{E} / \mathcal{G}_{E}^{+}}$contains $\sigma$. We need to show $\rho$ also contains $\operatorname{Ad}\left(g_{2}\right) \sigma$ (as a representation of $\mathcal{G}_{F_{2}} / \mathcal{G}_{F_{2}}^{+}$).

For convenience we shorten notation $F^{\prime}$ and $g^{\prime}$ for $F_{2}$ and $g_{2}$. Let $\mathcal{M}$ and $\mathcal{M}^{\prime}$ be Levi subgroups attached to $F$ and $F^{\prime}$ as in (5.1.1). The representation $\rho$ occurs in any irreducible principal series $\Pi=\operatorname{Ind}_{\mathcal{N} \mathcal{V}}^{\mathcal{G}}(\pi)$, where $\pi$ is an irreducible cuspidal representation of $\mathcal{M}$ containing $\sigma$. Here, $\mathcal{V}$ is a suitable unipotent radical. The principal series $\Pi$ can also be realized as $\operatorname{Ind}_{\mathcal{M}^{\prime} \mathcal{V}^{\prime}}^{\mathcal{G}}\left(\pi^{\prime}\right)$ where $\pi^{\prime}$ is an irreducible cuspidal representation containing $\operatorname{Ad}\left(g^{\prime}\right) \sigma$ of $\mathcal{M}_{g^{\prime} F}^{\prime} / \mathcal{M}_{g^{\prime} F}^{\prime+}=\mathcal{G}_{g^{\prime} F} / \mathcal{G}_{g^{\prime} F}^{+}$. We conclude $\rho$ occurs in $\operatorname{Ind}_{\mathcal{N}^{\prime} \mathcal{V}^{\prime}}^{\mathcal{G}}\left(\pi^{\prime}\right)$ from which we deduce that $\rho$ contains $\operatorname{Ad}\left(g^{\prime}\right) \sigma$.

Reversing the roles of $F_{1}$ and $F_{2}$ we see that any $\tau \in \widehat{\mathcal{G}_{E} / \mathcal{G}_{E}^{+}}$which contains $\operatorname{Ad}\left(g_{2}\right) \sigma$ also contains $\operatorname{Ad}\left(g_{1}\right) \sigma$. The lemma is shown.

We make the obvious definition that an associate class of cuspidal pairs is the collection of all cuspidal pairs which are associate to one another. It is an immediate consequence of Lemma 5.2 .2 that given an associate class $\mathcal{E}=\{(F, \sigma)\}$ of cuspidal pairs, the rule $B k=B k_{\mathcal{E}}$ which attaches to a facet $L \subset \mathcal{B}(\mathcal{G})$ the block or representations:

$(5.2 .3)$

$B k_{\mathcal{E}}(L)$

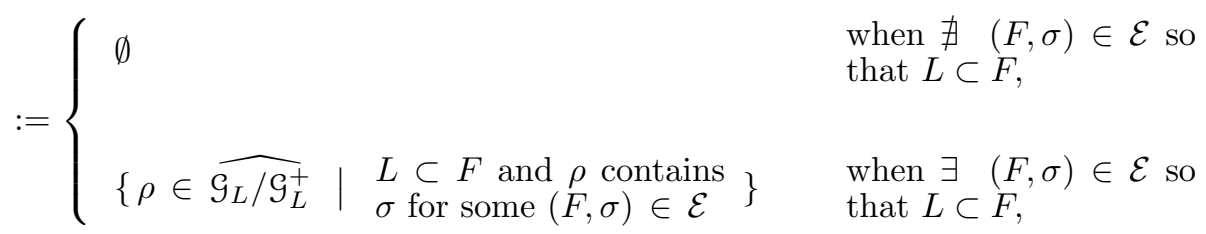

is $\mathcal{G}$-equivariant. To $\mathcal{E}$ and $L \subset \mathcal{B}$, we define the idempotent:

$$
e_{\mathcal{E}, L}:=\frac{1}{\#\left(\mathcal{G}_{L} / \mathcal{G}_{L}^{+}\right)} \sum_{\Theta_{\kappa} \in B k_{\mathcal{E}}(L)} \operatorname{deg}(\kappa) \Theta_{\kappa} \star e_{\mathcal{G}_{L}^{+}}
$$


We call this idempotent the Peter-Weyl idempotent attached to $\mathcal{E}$ and $L$. This clearly yields a $\mathcal{G}$-equivariant system of idempotents.

We note:

- Suppose $E$ is a facet associate to $F$, and facets $K, L$ satisfy $K \subset L \subset E$, i.e., $\mathcal{G}_{E} / \mathcal{G}_{K}^{+} \subset \mathcal{G}_{L} / \mathcal{G}_{K}^{+}$are parabolic subgroups of $\mathcal{G}_{K} / \mathcal{G}_{K}^{+}$. The finite field results say:

(i) $e_{\mathcal{E}, K} \star e_{\mathcal{G}_{L}^{+}}=e_{\mathcal{E}, L}$.

(ii) Under the hypothesis $K \subsetneq J \subset E$, i.e., $\mathcal{G}_{J} / \mathcal{G}_{K}^{+}$is a proper parabolic of $\mathcal{G}_{K} / \mathcal{G}_{K}^{+}$, with unipotent radical $\mathcal{G}_{J}^{+} / \mathcal{G}_{K}^{+} \neq\{1\}$; then

$$
\left(\sum_{K \subset L \subset E}(-1)^{\operatorname{dim}(L)} e_{\mathcal{E}, L}\right) \star e_{\mathcal{G}_{J}^{+}}=\text {zero function. }
$$

Theorem 5.2.5. Suppose $\mathcal{E}$ is the associate class of a cuspidal pair $(F, \sigma)$. Define idempotents as in (5.2.4). Then, the Euler-Poincaré sum

$$
P(\mathcal{E}):=\sum_{L \subset \mathcal{B}(\mathcal{G})}(-1)^{\operatorname{dim}(L)} e_{\mathcal{E}, L}
$$

over the facets of $\mathcal{B}(\mathcal{G})$ defines a $\mathcal{G}$-invariant essentially compact distribution.

Proof. The proof is an extension of the proof in the Iwahori setting. Fix a chamber $C_{0}$ and suppose $D$ is an arbitrary chamber. We take $\mathcal{S}$ to be a maximal split torus so that $\mathcal{A}(\mathcal{S})$ contains both chambers. Take $\Phi^{+} \subset \Phi(\mathcal{S})$ to be a set of positive roots so that $D \subset S\left(C_{0}, \Phi^{+}\right)$. As before, let $c(D)$, as in (2.3.7), denote the set of outward oriented faces of $D$ and define $D_{+}$and $\mathcal{F}_{+}(D)$ as in (4.2.2) and (4.2.3), i.e., $D_{+}=\bigcap_{F \in c(D)} F$ and $\mathcal{F}_{+}(D)$ is the set of facets which contain $D_{+}$. Suppose $J$ is an open compact subgroup. The key to adapting the Iwahori setting proof to the present one is to show

$$
\sum_{E \in \mathcal{F}_{+}(D)}(-1)^{\operatorname{dim}(E)} e_{\mathcal{E}, E} \star e_{J}=\text { zero function }
$$

when $\mathrm{ht}_{C_{0}}(D)$ is sufficiently large (dependent on $J$ ). We may and do assume the open compact subgroup $J$ has the form $J=\mathcal{G}_{x_{0}, \rho}$ for $x_{0} \in \operatorname{recess}\left(C_{0}\right)$, and $\rho$ is a positive integer.

- Define $R_{(\rho+2)}$ as in (4.4.1). For $y \in D \subset R_{(\rho+2)}$, we again have $\mathcal{G}_{x_{0}, \rho}$ contains the group $V_{y}$ of (4.4.3) and $V_{y} \mathcal{G}_{D_{+}}^{+}=\mathcal{G}_{D}^{+}$, so $V_{y} \mathcal{G}_{D_{+}}^{+} / \mathcal{G}_{D_{+}}^{+}$is the unipotent radical of the Borel subgroup $\mathcal{G}_{D} / \mathcal{G}_{D_{+}}^{+}$of $\mathcal{G}_{D_{+}} / \mathcal{G}_{D_{+}}^{+}$. The analogue of the computations (4.4.4) and (4.4.5) are

$$
\sum_{E \in \mathcal{F}_{+}(D)}(-1)^{\operatorname{dim}(E)} e_{\mathcal{E}, E} \star e_{V_{y}}=\text { zero function }
$$

and

$$
\sum_{E \in \mathcal{F}_{+}(D)}(-1)^{\operatorname{dim}(E)} e_{\mathcal{E}, E} \star e_{\mathcal{G}_{x_{0}, \rho}}=\text { zero function . }
$$

A difference between the Iwahori setting and the general depth zero setting is the following: In the Iwahori setting, the individual convolution terms $e_{E} \star e_{V}$ are all non-zero, but their alternating sum is zero. In the general setting, some of the individual convolutions $e_{\mathcal{E}, E} \star e_{V}$ are zero due to the cuspidal assumption on $\mathcal{E}$. 
- We again use the sets in (4.4.6) to partition $R_{(\rho+2)}$. Fix $I \subset \Delta$. With $V_{y}$ $(y \in I)$ defined as in (4.4.7), we have $V_{y} \mathcal{G}_{D_{+}}^{+} / \mathcal{G}_{D_{+}}^{+}$is the unipotent radical of a parabolic subgroup of $\mathcal{G}_{D_{+}} / \mathcal{G}_{D_{+}}^{+}$, i.e., there is a $K \in \mathcal{F}_{+}(D)$ so that $V_{y} \mathcal{G}_{D_{+}}^{+}=\mathcal{G}_{K}^{+}$. With finitely many exceptions, the unipotent radical is not $\{1\}$, and so Corollary 3.2.16 applies to give

$$
\sum_{E \in \mathcal{F}_{+}(D)}(-1)^{\operatorname{dim}(E)} e_{\tau, E} \star e_{V_{y}}
$$

is the zero function, and therefore

$$
\sum_{E \in \mathcal{F}_{+}(D)}(-1)^{\operatorname{dim}(E)} e_{\tau, E} \star e_{\mathcal{G}_{x_{0}, \rho}}
$$

vanishes too. We handle the finite number of exceptions by replacing $(\rho+2)$ by a larger value to exclude these finitely many exceptions. Again, some of the convolutions $e_{\tau, E} \star e_{\mathcal{G}_{x_{0}, \rho}}$ vanish due to the cuspidality of $\tau$. The theorem follows.

Corollary 5.2.6. The distribution $P(\mathcal{E})$ is the Bernstein projector $P(F, \sigma)$ of (5.2.1).

Proof. We replace the facet $F$ by an associate one in the 'base' chamber $C_{0}$. Then, as extensions of the Iwahori situation we have:

$$
\sum_{E \subset C_{0}}(-1)^{\operatorname{dim}(E)} e_{\tau, E} \star e_{\mathcal{G}_{F}^{+}}=e_{\tau, F} .
$$

(ii) For any chamber $D \neq C_{0}$ :

$$
\sum_{E \subset \mathcal{F}_{+}(D)}(-1)^{\operatorname{dim}(E)} e_{\tau, E} \star e_{\mathcal{G}_{F}^{+}}=\text {zero function . }
$$

(iii)

$$
\left(\sum_{E \subset \mathcal{B}(\mathcal{G})}(-1)^{\operatorname{dim}(E)} e_{\tau, E}\right) \star e_{\mathcal{G}_{F}^{+}}=e_{\tau, F} .
$$

Set $P=P(\mathcal{E})=\left(\sum_{E \subset \mathcal{B}(\mathcal{G})}(-1)^{\operatorname{dim}(E)} e_{\tau, E}\right)$. If $\left(\pi, V_{\pi}\right)$ is an irreducible smooth representation of $\mathcal{G}$, by (iii), we have

$$
\pi\left(e_{\tau, F}\right)=\pi\left(P \star e_{\mathcal{G}_{F}^{+}}\right)=\pi(P) \pi\left(e_{\mathcal{G}_{F}^{+}}\right) .
$$

When $\pi$ belongs to a Bernstein component $\left(\mathcal{M}, \mathrm{c}-\operatorname{Ind}_{\mathcal{F}_{F}}^{\mathcal{M}}(\tau)\right)$ the left size is non-zero and we conclude $\pi(P)$ is the identity. Additionally, the fact that the individual terms of $P$ have the form $e_{\tau, E}$ means $\pi(P)$ is zero unless there exists a facet $E$ so that $\pi\left(e_{\tau, E}\right)$ is non-zero. Hence, $P$ is the Bernstein projector.

5.3. The depth zero projector. When we sum over all the Bernstein components of depth zero we obtain :

- $P_{0}=\sum_{\rho(\Omega)=0} P(\Omega)$, which is, by definition, the depth zero projector.

- For any facet $F$, the sum of the Peter-Weyl idempotents is $\frac{1}{\text { meas }\left(\mathcal{G}_{F}\right)}$ times the character of the regular representation of $\mathcal{G}_{F} / \mathcal{G}_{F}^{+}$. This is the idempotent $e_{\mathcal{G}_{F}^{+}}=\frac{1}{\operatorname{meas}\left(\mathcal{G}_{F}^{+}\right)} 1_{\mathcal{G}_{F}^{+}}$.

The resulting Euler-Poincaré formula for $P_{0}$ is exactly the one in $[\mathrm{BKV}]$. 


\section{NON-SPLIT GROUPS}

In this section, we explain the modifications needed in the proofs of sections 4 and 5 so that they apply when the $k$-defined group $\mathbf{G}$ is non-split. We assume $\mathbf{G}$ is connected, absolutely quasisimple. Set $\mathcal{G}=\mathbf{G}(k)$. Let $K$ be the maximal unramified extension of $k$, and let $\operatorname{Gal}(K / k)$ denote the Galois group.

6.1. We recall (see $[\mathrm{T}, \S 1.10]$ ) there exists a torus $\mathrm{S}$ defined over $k$ satisfying: (i) $\mathbf{S}$ is a maximal split $K$-torus, and (ii) $\mathbf{S}:=\mathrm{S}^{\mathrm{Gal}(K / k)}$ is a maximal split $k$-torus. We also recall the result of Steinberg that $\mathbf{G}$ is quasisplit over $K$, and therefore, the centralizer $\mathbf{Z}=C_{\mathbf{G}}(\mathrm{S})$ is a maximal $k$-torus. Furthermore:

- Since $\mathbf{G}$ is assumed to be absolutely quasisimple, the Bruhat-Tits building $\mathcal{B}(\mathbf{G}(K))$ is a simplicial complex, and both $\mathbf{G}(K)$ and $\mathrm{Gal}(K / k)$ act by simplicial automorphisms. The building $\mathcal{B}(\mathcal{G})$ is the $\operatorname{Gal}(K / k)$-fixed points of $\mathcal{B}(\mathbf{G}(K))$.

- Let $\mathcal{A}(\mathbf{S}(K))$ be the apartment of $\mathbf{S}(K)$, and let $\Psi(\mathbf{S}(K))$ be the corresponding system of affine roots. The fixed points $\mathcal{A}(\mathbf{S}(K))^{\operatorname{Gal}(K / k)}$ and $\mathcal{B}(\mathbf{G}(K))^{\operatorname{Gal}(K / k)}$ are identified (defined) as the building $\mathcal{B}(\mathcal{G})$ and apartment $\mathcal{A}(\mathcal{S}) \quad\left(\mathcal{S}=\mathbf{S}(k)=\mathbf{S}(K)^{\mathrm{Gal}(K / k)}\right)$. Let $\mathbf{M}_{\mathrm{o}}=C_{\mathbf{G}}(\mathbf{S})$, a minimal Levi $k$-subgroup. Given a choice of positive roots $\Phi^{+}$, let $\mathbf{P}_{\mathrm{o}}=\mathbf{M}_{\mathrm{o}} \mathbf{U}_{\mathrm{o}}$ be the corresponding minimal parabolic $k$-subgroup. We set $\mathcal{M}_{\mathrm{o}}=\mathbf{M}_{\mathrm{o}}(k)$, $\mathcal{U}_{\mathrm{o}}=\mathbf{U}_{\mathrm{o}}(k)$, and $\mathcal{P}_{\mathrm{o}}=\mathcal{M}_{\mathrm{o}} \mathcal{U}_{\mathrm{o}}=\mathbf{P}_{\mathrm{o}}(k)$.

- $\quad$ The affine root system $\Psi=\Psi(\mathcal{S})$ on $\mathcal{A}$ consists of all non-constant restrictions to $\mathcal{A}$ of affine roots in $\Psi(\mathrm{S}(K)$ ) (see [T, $\S 1.10 .1]$ ). If $E$ is a facet of $\mathcal{B}$, and $x, y \in \operatorname{recess}(E)$, then

$$
\mathcal{G}_{x, 0}=\mathcal{G}_{y, 0} \quad \text { and } \quad \mathcal{G}_{x, 0^{+}}=\mathcal{G}_{y, 0^{+}} .
$$

Because of these equalities, we denote the common subgroups as $\mathcal{G}_{E}$ and $\mathcal{G}_{E}^{+}$. We note that if $F$ is a subfacet of $E$, then

$$
\mathcal{G}_{F} \supset \mathcal{G}_{E} \supset \mathcal{G}_{E}^{+} \supset \mathcal{G}_{F}^{+},
$$

and $\mathcal{G}_{E} / \mathcal{G}_{F}^{+}$is a parabolic subgroup of $\mathcal{G}_{F} / \mathcal{G}_{F}^{+}$.

- $\quad$ Let $z=Z(k)$, and let $z_{c}$ be the maximal bounded (compact) subgroup of $z$. The group $N_{\mathcal{G}}(z)$ acts as orthogonal affine maps on $\mathcal{A}$ with $z_{c}$ acting trivially. We call $N_{\mathcal{G}}(\mathcal{Z}) / z_{c}$ the extended affine Weyl group of $\mathcal{G}$. The Coxeter group $\mathrm{W}_{\Psi}$ of $\Psi(\mathcal{A})$, i.e., the symmetry group of $\mathcal{A}$ generated by reflections in the hyperplanes $H_{\psi},(\psi \in \Psi)$ is a finite index subgroup of $N_{\mathcal{G}}(\mathcal{Z}) / \mathcal{Z}_{c}$. The action of $\mathrm{W}_{\Psi}$ is transitive on the chambers of $\mathcal{A}$ (see [T, §1.8]). For $x \in \mathcal{A}$, define

$$
\mathrm{W}_{x}:=\left\{\begin{array}{l}
\text { symmetry group of } \mathcal{A} \text { generated by reflections across affine hyper- } \\
\text { planes } H_{\psi} \text { containing } x, \text { i.e., } \psi(x)=0 .
\end{array}\right.
$$

Set $\Phi:=\{\operatorname{grad}(\psi) \mid \psi \in \Psi\}$, a possibly non-reduced root system, and let $\mathrm{W}_{\Phi}$ denote the Coxeter group of $\Phi$. We recall a point $x$ is called special if $W_{x}$ and $W_{\Phi}$ are the same. Special points always exist (see [T, §1.9]). When $x$ is special, the group $N_{\mathcal{G}}(z) / z_{0}$ is the semidirect product of the group $W_{x}$ and the normal subgroup of translations $X=z / z_{c}$. Similarly, $W_{\Psi}$ is a semidirect product of the group of $W_{x}$ and translation subgroup of $W_{\Psi}$. 
Fix a chamber $C_{0} \subset \mathcal{A}$ which contains $x$. For $\alpha \in \Phi$, let $S(\alpha)$ be the set of roots in $\Phi$ which are a multiple of $\alpha$. Trivially, the sets $S(\alpha)$ partition $\Phi$. As in (2.3.4), define

$$
\mathrm{ht}_{C_{0}}^{S(\alpha)}(D):=\left\{\begin{array}{l}
\text { The number of affine hyperplanes } H_{\psi} \text { satisfying: } \\
\text { (i) } \operatorname{grad}(\psi) \in S(\alpha) \text {, and } \\
\text { (ii) } H_{\psi} \text { separates } C_{0} \text { and } D .
\end{array}\right.
$$

- The obvious analogue of Lemma 2.3.5 holds. Indeed, by [BTa, §1.4], there is a canonical reduced root system $\Sigma_{\mathrm{o}} \subset \Phi$ so that the affine root system

$$
\Sigma_{\text {aff }}:=\left\{\alpha+k \mid \alpha \in \Sigma_{\mathrm{o}}, k \in \mathbb{Z}\right\}
$$

has associated affine hyperplanes $H_{\psi}, \psi \in \Sigma_{\text {aff }}$ exactly the same as the affine hyperplanes of $\Psi$. In particular, the affine Weyl groups of the affine root systems $\Sigma_{\text {aff }}$ and $\Psi$ are the same.

- The proof of Proposition 2.5.5used only the affine hyperplanes $H_{ \pm \psi}$, and it is valid in the non-split situation with $\Psi$ replaced by $\Sigma_{\text {aff }}$; so, Proposition 2.5.5 holds. We use (2.4.2) to define the $C_{0}$-based sector $S\left(C_{0}, \Phi^{+}\right)$.

- The results of $\mathrm{MPb}$ stated in section 5 hold in the non-split situation.

Theorem 6.1.3. Suppose $\mathbf{G}$ is a connected absolutely quasisimple $k$-group. Let $\mathcal{G}=\mathbf{G}(k)$, and let $\mathcal{B}=\mathcal{B}(\mathcal{G})$ be the Bruhat-Tits building. Suppose $(F, \sigma)$ is a cuspidal pair and $\mathcal{E}$ the associate class of $(F, \sigma)$. Define a $\mathcal{G}$-equivariant system of idempotents as in (5.2.4). Then,

- The Euler-Poincaré sum

$$
P(\mathcal{E})=\sum_{L \subset \mathcal{B}(\mathcal{G})}(-1)^{\operatorname{dim}(L)} e_{\tau, L}
$$

over the facets of $\mathcal{B}(\mathcal{G})$ defines a $\mathcal{G}$-invariant essentially compact distribution.

- With Levi subgroup $\mathcal{M}$ defined as in (5.1.1), the distribution $P(\mathcal{E})$ is the Bernstein projector $P(F, \sigma)$ of (5.2.1).

Proof. The proof of Theorem 6.1.3 is adapted from those of Theorems 4.2.4 and 4.2.1. We fix a chamber $C_{0}$. Suppose $D\left(\neq C_{0}\right)$ is any other chamber. For an arbitrary fixed open compact subgroup $J$ we need to show $P \star e_{J} \in C_{c}^{\infty}(\mathcal{G})$. It suffices to show

$$
\sum_{E \in \mathcal{F}_{+}(D)}(-1)^{\operatorname{dim}(E)} e_{\tau, E} \star e_{J}=\text { zero function }
$$

when $\mathrm{ht}_{C_{0}}(D)$ is sufficiently large. Fix $x_{\mathrm{o}} \in C_{0}$, and take $\rho \in \mathbb{R}_{>0}$ sufficiently large so that $\mathcal{G}_{x_{\mathrm{o}}, \rho} \subset J$. We replace $J$ with $\mathcal{G}_{x_{\mathrm{o}}, \rho}$. Take $\mathbf{S} \supset \mathbf{S}\left(\mathbf{S}=\mathbf{S}^{\operatorname{Gal}(K / k)}\right)$, as above, so that $D$ is in $\mathcal{A}=\mathcal{A}(\mathcal{S})$, and take a positive system of roots $\Phi^{+} \subset \Phi(\mathcal{S})$, so that $S\left(C_{0}, \Phi^{+}\right)$contains $D$. Let $N$ be a sufficiently large integer so that for any simple root $\alpha \in \Delta\left(\left(\Phi^{\text {red }}\right)^{+}\right)$, when $C_{0}$ and $D$ are separated by $N$ affine hyperplanes perpendicular to $\alpha$, then:

$\forall \psi \in \Psi=\Psi(\mathcal{S})$, satisfying $\lambda_{\alpha}(\operatorname{grad}(\psi))>0$, i.e., when $\operatorname{grad}(\psi)$ is expressed as a linear (non-negative) combination of simple roots the $\alpha$ coefficient is non-zero, then $\forall x \in C_{0}$ and $\forall y \in D$, we have $(\psi(y)-\psi(x))>\rho$. 
If such a $\psi$ vanishes on $D_{+}$, and we take $y \in D_{+}$, we see $-\psi(x)=(\psi(y)-\psi(x))$ $>\rho$, This means $\left(\mathcal{G}_{x, \rho} \cap \mathcal{U}_{-\gamma}\right) \supset \mathcal{X}_{-\psi}$, where $\gamma=\operatorname{grad}(\psi)$. We note in the nonsplit situation, the root group $\mathcal{U}_{-\gamma}$ and the affine root group $\mathcal{X}_{-\psi}$ may be noncommutative. Define $R_{N}$ as in (4.4.1); roughly the set of chambers which are Bruhat distance at least $N$ from the walls of $S\left(C_{0}, \Phi^{+}\right)$. The above says for any chamber $D$ in $\mathcal{R}_{N}$, the subgroup $\mathcal{G}_{x, \rho}$ contains the subgroup

$$
V:=\prod_{\psi \in \Psi\left(D_{+}, \Phi^{+}\right)} x_{-\psi},
$$

and $V \mathcal{G}_{D_{+}}^{+} / \mathcal{G}_{D_{+}}^{+} \subset \mathcal{G}_{D_{+}} / \mathcal{G}_{D_{+}}^{+}$is the unipotent radical of the Borel subgroup $\mathcal{G}_{D_{+}} / \mathcal{G}_{D_{+}}^{+}$. We then have the analogue of (4.4.4) and (4.4.5), i.e.,

$$
\sum_{E \in \mathcal{F}_{+}(D)}(-1)^{\operatorname{dim}(E)} e_{E} \star e_{\mathcal{G}_{x_{0}, \rho}}=\text { zero function } .
$$

The situation when $D$ is a chamber in $S\left(C_{0}, \Phi^{+}\right) \backslash R_{N}$ is handled by defining sets $R_{\{I, k\}}$ as in (4.4.6), and adapting the argument. We omit the very similar details. This completes the proof that the Euler-Poincaré sum (6.1.4) defines a Bernstein center distribution.

To establish that $P$ is the projector, we use Corollary 3.2 .15 and adapt the proof of Corollary 4.5 .2 to deduce :

$$
\sum_{E \subset C_{0}}(-1)^{\operatorname{dim}(E)} e_{\tau, E} \star e_{\mathcal{G}_{F}^{+}}=e_{\tau, F}
$$

For any chamber $D \neq C_{0}$ :

$$
\sum_{E \subset \mathcal{F}_{+}(D)}(-1)^{\operatorname{dim}(E)} e_{\tau, E} \star e_{\mathcal{G}_{F}^{+}}=\text {zero function . }
$$

That $P$ is the projector then follows.

\section{ACKNOWLEDGMENTS}

Parts of this work were done during visits by the first and third authors to the University of Utah Mathematics Department in summer, 2015, by the first author to the HKUST Mathematics Department in summer, 2016, and by the third author to the University of Oxford Mathematics Department in summer, 2016. The departments are thanked for their hospitality. The referees are thanked for their corrections and useful comments.

\section{REFERENCES}

[B] J. Bernstein, Notes of lectures on Representations of p-adic Groups, Harvard University, Fall 1992, written by K. E. Rumelhart.

[BD] J. N. Bernstein, Le "centre" de Bernstein (French), Representations of reductive groups over a local field, Travaux en Cours, Hermann, Paris, 1984, pp. 1-32. Edited by P. Deligne. MR.771671

[BKV] Roman Bezrukavnikov, David Kazhdan, and Yakov Varshavsky, On the depth $r$ Bernstein projector, Selecta Math. (N.S.) 22 (2016), no. 4, 2271-2311, DOI 10.1007/s00029-016-02782. MR3573958

[BTa] F. Bruhat and J. Tits, Groupes réductifs sur un corps local (French), Inst. Hautes Études Sci. Publ. Math. 41 (1972), 5-251. MR0327923

[BTb] F. Bruhat and J. Tits, Groupes réductifs sur un corps local (French), Inst. Hautes Études Sci. Publ. Math. 41 (1972), 5-251. MR0327923 
[Dt] J.-F. Dat, Quelques propriétés des idempotents centraux des groupes p-adiques (French, with English summary), J. Reine Angew. Math. 554 (2003), 69-103, DOI 10.1515/crll.2003.009. MR.1952169

[Dn] Pierre Deligne, Le support du caractère d'une représentation supercuspidale (French, with English summary), C. R. Acad. Sci. Paris Sér. A-B 283 (1976), no. 4, Aii, A155-A157. MR 0425033

[HC] Harish-Chandra, Eisenstein series over finite fields, Functional analysis and related fields (Proc. Conf. M. Stone, Univ. Chicago, Chicago, Ill., 1968), Springer, New York, 1970, pp. 76-88. MR0457579

[IM] N. Iwahori and H. Matsumoto, On some Bruhat decomposition and the structure of the Hecke rings of $\mathfrak{p}$-adic Chevalley groups, Inst. Hautes Études Sci. Publ. Math. 25 (1965), 5-48. MR 0185016

[K] Robert E. Kottwitz, Tamagawa numbers, Ann. of Math. (2) 127 (1988), no. 3, 629-646, DOI 10.2307/2007007. MR942522

[MPa] Allen Moy and Gopal Prasad, Unrefined minimal K-types for p-adic groups, Invent. Math. 116 (1994), no. 1-3, 393-408, DOI 10.1007/BF01231566. MR1253198

[MPb] Allen Moy and Gopal Prasad, Jacquet functors and unrefined minimal $K$-types, Comment. Math. Helv. 71 (1996), no. 1, 98-121, DOI 10.1007/BF02566411. MR1371680

[MS] Ralf Meyer and Maarten Solleveld, Resolutions for representations of reductive $p$ adic groups via their buildings, J. Reine Angew. Math. 647 (2010), 115-150, DOI 10.1515/CRELLE.2010.075. MR.2729360

[SS] Peter Schneider and Ulrich Stuhler, Representation theory and sheaves on the Bruhat-Tits building, Inst. Hautes Études Sci. Publ. Math. 85 (1997), 97-191. MR1471867

[T] J. Tits, Reductive groups over local fields, Automorphic forms, representations and $L$ functions (Proc. Sympos. Pure Math., Oregon State Univ., Corvallis, Ore., 1977), Proc. Sympos. Pure Math., XXXIII, Amer. Math. Soc., Providence, R.I., 1979, pp. 29-69. MR.546588

Department of Mathematics, Malott Hall, Cornell University, Ithaca, New York 14853-0099

Email address: barbasch@math.cornell.edu

Mathematical Institute, Andrew Wiles Bullding, University of Oxford, Oxford, OX2 6GG, UNITED KINGDOM

Email address: dan.ciubotaru@maths.ox.ac.uk

Department of Mathematics, The Hong Kong University of Science and Technology, Clear Water Bay Road, Hong Kong

Email address: amoy@ust.hk 